\title{
Sharp Bounds of First Zagreb Coindex for F-Sum Graphs
}

\author{
Muhammad Javaid $\left(\mathbb{D},{ }^{1}\right.$ Muhammad Ibraheem, ${ }^{1}$ Uzma Ahmad $\mathbb{D},{ }^{2}$ and Jia-Bao Liu $\mathbb{D}^{3}$ \\ ${ }^{1}$ Department of Mathematics, School of Science, University of Management and Technology, Lahore 54770, Pakistan \\ ${ }^{2}$ Department of Mathematics, University of the Punjab, Lahore, Pakistan \\ ${ }^{3}$ School of Mathematics and Physics, Anhui Jianzhu University, Hefei 230601, China \\ Correspondence should be addressed to Jia-Bao Liu; liujiabaoad@163.com
}

Received 26 March 2021; Accepted 11 September 2021; Published 13 October 2021

Academic Editor: M.M. Bhatti

Copyright (c) 2021 Muhammad Javaid et al. This is an open access article distributed under the Creative Commons Attribution License, which permits unrestricted use, distribution, and reproduction in any medium, provided the original work is properly cited.

\begin{abstract}
Let $G=(V(E), E(G))$ be a connected graph with vertex set $V(G)$ and edge set $E(G)$. For a graph $G$, the graphs $S(G), R(G), Q(G)$, and $T(G)$ are obtained by applying the four subdivisions related operations $S, R, Q$, and $T$, respectively. Further, for two connected graphs $G_{1}$ and $G_{2}, G_{1+F} G_{2}$ are $F$-sum graphs which are constructed with the help of Cartesian product of $F\left(G_{1}\right)$ and $G_{2}$, where $F \in\{S, R, Q, T\}$. In this paper, we compute the lower and upper bounds for the first Zagreb coindex of these $F$-sum ( $S$-sum, $R$-sum, $Q$-sum, and T-sum) graphs in the form of the first Zagreb indices and coincides of their basic graphs. At the end, we use linear regression modeling to find the best correlation among the obtained results for the thirteen physicochemical properties of the molecular structures such as boiling point, density, heat capacity at constant pressure, entropy, heat capacity at constant time, enthalpy of vaporization, acentric factor, standard enthalpy of vaporization, enthalpy of formation, octanol-water partition coefficient, standard enthalpy of formation, total surface area, and molar volume.
\end{abstract}

\section{Introduction}

The subject of graph theory is growing and moving into mainstream of mathematics, playing a basic role in various disciplines of science especially in chemistry and computer science. A topological index (TI) is a function from a set of graphs to the set of real numbers that assigns a different numerical value to each graph unless the graphs are isomorphic. Moreover, this numeric value predicts various physical and chemical properties of the involved organic compounds in the molecular graphs such as volume, density, pressure, weight, boiling point, freezing point, vaporization point, heat of formation, and heat of evaporation [1,2]. TIs are also used to study the quantitative structure-activity relationship (QSAR) and quantitative structure-property relationship (QSPR) and medical behaviors of different drugs in the subject of cheminformatics and pharmaceutical industries, respectively [3-5].

There are various types of TIs based on degree, distance, and polynomial, but degree-based TIs are more studied than others (see the latest survey [6]). Wiener calculated boiling point of paraffin by using a distance-based TI (see [7]). Gutman and Trinajsti defined the TIs known as first and second Zagreb indices to calculate total $\pi$-electron energy of alternant hydrocarbons [8].

There are different operations on graphs such as joining, deleting, union, intersection, product, complement, switching, and subdivision. These operations on graphs play an important role to obtain the new molecular graphs from the old ones. Yan et al. listed five graphs $L(G)$, $S(G), Q(G), R(G)$, and $T(G)$ with the help of five operations $L, S, Q, R$, and $T$, respectively. They also studied the behavior of Wiener index of graphs under these five decorations (see [4]). Eliasi and Taeri introduced the four subdivision-related operations using the concept of Cartesian product and obtained four sum graphs $\left(G_{1+S} G_{2}\right.$, $G_{1+R} G_{2}, G_{1+Q} G_{2}$, and $\left.G_{1+T} G_{2}\right)$. They also computed the Wiener indices of these newly defined $F$-sum graphs (see [9]). Akhtar and Imran calculated the forgotten index [10], Deng et al. [11] computed first and second Zagreb indices, and Liu et al. computed first general Zagreb index of these graphs (see [12]). 
Ashrafi et al. computed Zagreb coindices of composite graph operations such as joining, union, disjunction, Cartesian product, corona product, and composition (see [13]). Mansour and Song computed $a$ and $(a, b)$-analogs of Zagreb indices and coindices of graphs [14]. Ashrafi et al. worked on extremal graphs with respect to the Zagreb coindices [15]. Ramane et al. calculated coindices for the transmission- and reciprocal transmission-based graphs (see [16]). Javaid et al. computed the first Zagreb connection index and coindex of some derived graphs [17].

In this paper, we compute the lower and upper bounds for the first Zagreb coindex of $F$-sum ( $S$-sum, $R$-sum, $Q$-sum, and $T$-sum) graphs in the form of the first Zagreb indices and coincides of their basic graphs. At the end, the obtained results are also illustrated with the help of the examples of the exact and bonded values for some particular F-sum graphs. The reset of the paper is organized as follows: Section 2 includes the basic definitions and notions, Section 3 contains the main results of our work, and Section 4 presents particular examples related to the main results.

\section{Preliminaries}

Let $G=(V(G), E(G))$ be a connected graph, where $|V(G)|$ is order and $|E(G)|$ is size of the graph $G$. For any vertex $v \in V(G)$, its degree is denoted by $d(v)$ and is defined as number of vertices incident on it. An edge is formed by joining of two vertices $u, v \in V(G)$ denoted by $u v$. The complement of any graph is denoted by $\bar{G}$ and is defined as having same number of vertices as of original graph $G$ but for any two vertices $u$ and $v, u v \in E(\bar{G})$ iff $u v \notin E(G)$. The first and second Zagreb indices $M_{1}(G)$ and $M_{2}(G)$ of $G$ are defined in [8] as follows:

$$
\begin{aligned}
& M_{1}(G)=\sum_{t \in V(G)}\left[d_{G}(t)\right]^{2}=\sum_{t_{1} t_{2} \in E(G)}\left[d_{G}\left(t_{1}\right)+d_{G}\left(t_{2}\right)\right], \\
& M_{2}(G)=\sum_{t_{1} t_{2} \in E(G)}\left[d_{G}\left(t_{1}\right) d_{G}\left(t_{2}\right)\right] .
\end{aligned}
$$

The first Zagreb coindex $\bar{M}_{1}(G)$ is defined in [15] as follows:

$$
\bar{M}_{1}(G)=\sum_{t_{1} t_{2} \notin E(G)}\left[d_{G}\left(t_{1}\right)+d_{G}\left(t_{2}\right)\right] .
$$

It should be noted that the Zagreb coindices of $G$ run over $E(\bar{G})$, but the degrees correspond to $G$. For two connected graphs $G_{1}$ and $G_{2}$, the $F$-sum graphs are defined as follows.

Let $G$ be a graph; then,

(i) $S(G)$ is a graph formed by putting one vertex in each edge of $G$.

(ii) $R(G)$ is a graph obtained from $S(G)$ by joining the adjacent vertices of $G$.

(iii) $Q(G)$ is a graph formed from $S(G)$ by joining the pairs of new vertices which are on the adjacent edges of $G$.

(iv) $T(G)$ is formed by applying both operations of $R(G)$ and $Q(G)$ on $S(G)$.

Suppose that $G_{1}$ and $G_{2}$ are two connected graphs; then, their F-sum graphs are denoted by $G_{1+F} G_{2}$ and defined with vertex set $\left|V\left(G_{1+S} G_{2}\right)\right|=V\left(G_{1}\right) \cup E\left(G_{1}\right) \times V\left(G_{2}\right)$ and edge set as the vertices $\left(u_{1}, u_{2}\right)$ and $\left(v_{1}, v_{2}\right)$ of $G_{1+F} G_{2}$ are joined iff

(i) $u_{1}=v_{1} \in V\left(G_{1}\right)$ and $u_{2} \backsim v_{2} \in G_{2}$.

(ii) $u_{2}=v_{2} \in V\left(G_{2}\right) \quad$ and $\quad u_{1} \backsim v_{1} \in F\left(G_{1}\right)$, where $F \in\{S, R, Q, T\}$.

For details, see Figures 1 and 2 .

\section{Main Results}

In this section, we discussed the main results of the first Zagreb coindex on the $F$-sum graphs which will be denoted by simply $M^{-}$.

Theorem 1. Let $G_{1}$ and $G_{2}$ be two connected graphs; then, first Zagreb coindex of $G_{1+S} G_{2}$ is given as

$$
\alpha_{1} \leq \bar{M}\left(G_{1+s} G_{2}\right) \leq \alpha_{2} \text {, }
$$

where

$$
\begin{aligned}
\alpha_{1}= & 2\left(n_{2}^{2} e_{1}^{2}-n_{2} e_{1}\right)+2\left(e_{1} \overline{e_{2}}+e_{2}\left(e_{1}+\overline{e_{1}}\right)\right)+n_{2} e_{1}\left(n_{1}-2\right) \\
& \cdot\left(e_{2}+n_{2}\right)+\left(2\left(e_{2}+\bar{e}_{2}\right)+n_{2}\right) M_{1}\left(G_{1}\right)+\left(n_{2}+2 e_{2}+2 \bar{e}_{2}\right) \bar{M}_{1}\left(G_{1}\right) \\
& +\left(2 e_{2}+2 \bar{e}_{1}\right) M_{1}\left(G_{2}\right)+n_{1}+2\left(\bar{e}_{1}+e_{1}\right) \bar{M}_{1}\left(G_{2}\right)+2 n_{2} e_{1}\left[\left(2 n_{2}-1\right)+2\left(n_{2}-1\right)\left(e_{2}+n_{2}\right)\right] \\
\alpha_{2}= & 2\left(n_{2}^{2} e_{1}^{2}-n_{2} e_{1}\right)+2\left(e_{1} \overline{e_{2}}+e_{2}\left(e_{1}+\overline{e_{1}}\right)\right)+n_{2} e_{1}\left(n_{1}-2\right) \\
& \cdot\left(e_{2}+n_{2}\right)+\left(2\left(e_{2}+\bar{e}_{2}\right)+n_{2}\right) M_{1}\left(G_{1}\right)+\left(n_{2}+2 e_{2}+2 \bar{e}_{2}\right) \bar{M}_{1}\left(G_{1}\right) \\
& +\left(2 e_{2}+2 \bar{e}_{1}\right) M_{1}\left(G_{2}\right)+\left(n_{1}+2\left(\bar{e}_{1}+e_{1}\right)\right) \bar{M}_{1}\left(G_{2}\right)+2 e_{1} E\left(S\left(G_{1}\right)\right)\left[\left(n_{1}-2\right) n_{2}\left(2 n_{2}-1\right)+2\left(n_{2}-1\right)\left(e_{2}+n_{2}\right)\right] .
\end{aligned}
$$



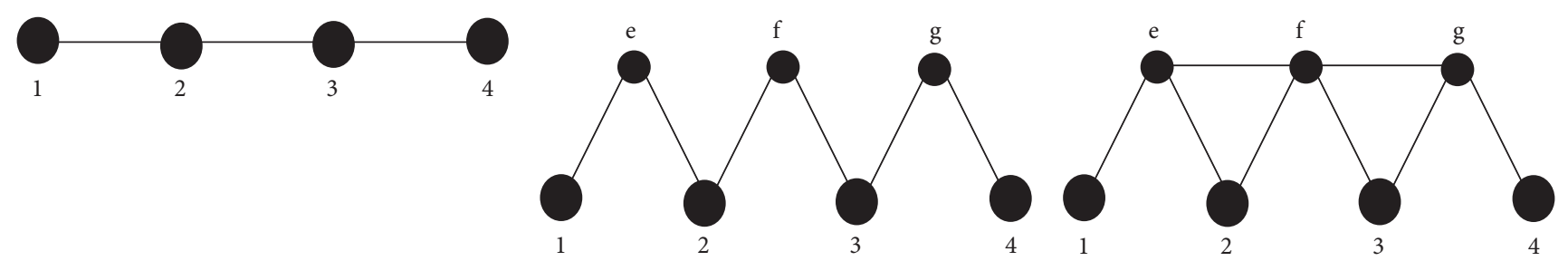

(a)

(b)

(c)

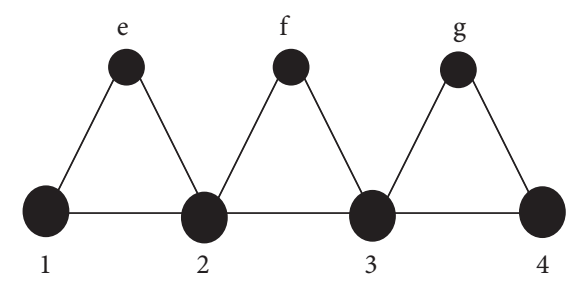

(d)

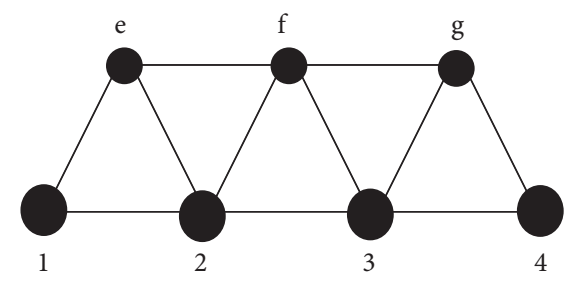

(e)

Figure 1: (a) $G \cong P_{4}$, (b) $S(G) \cong S\left(P_{4}\right)$, (c) $Q(G) \cong\left(P_{4}\right)$, (d) $R(G) \cong R\left(P_{4}\right)$, and (e) $T(G) \cong T\left(P_{4}\right)$.

Proof. Using equation (2), we have

$$
\begin{aligned}
& \bar{M}\left(G_{1+S} G_{2}\right)=\sum_{\left(t_{1}, t_{2}\right)} \sum_{\left(x_{1}, x_{2}\right) \notin E\left(G_{1+S} G_{2}\right)}\left[d\left(t_{1}, x_{1}\right)+d\left(t_{2}, x_{2}\right)\right] a, \\
& \bar{M}\left(G_{1+S} G_{2}\right)=\sum_{t_{1}, t_{2} \in\left(V\left(S G_{1}\right)-V\left(G_{1}\right)\right)} \sum_{x_{1}, x_{2} \in V_{G_{2}}}\left[d\left(t_{1}, x_{1}\right)+d\left(t_{2}, x_{2}\right)\right] \\
& +\sum_{t_{1}, t_{2} \in V_{G_{1}},} \sum_{x_{1}, x_{2} \in V_{G_{2}}}\left[d\left(t_{1}, x_{1}\right)+d\left(t_{2}, x_{2}\right)\right] \\
& \sum_{\substack{t_{1}, t_{2} \in V\left(S\left(G_{1}\right)\right) \\
t_{1} \in V\left(G_{1}\right) \\
t_{2} \in V\left(S\left(G_{1}\right)-V\left(G_{1}\right)\right)}} \sum_{x_{1}, x_{2} \in V_{G_{2}}}\left[d\left(t_{1}, x_{1}\right)+d\left(t_{2}, x_{2}\right)\right], \\
& =\sum A+\sum B+\sum C, \\
& \sum A=\sum_{t_{1}, t_{2} \in V} \sum_{\left.S\left(G_{1}\right)-V\left(G_{1}\right)\right)}\left[d_{S\left(G_{1}\right)}\left(t_{1}\right)+d_{S\left(G_{1}\right)}\left(t_{2}\right)\right] \\
& =\sum_{t_{1}, t_{2} \in V\left(S\left(G_{1}\right)-V\left(G_{1}\right)\right)} \sum_{x_{1}, x_{2} \in V_{G_{2}}}(2+2)=2\left(n_{2}^{2} e_{1}^{2}-n_{2} e_{1}\right), \\
& \sum A=2\left(n_{2}^{2} e_{1}^{2}-n_{2} e_{1}\right)
\end{aligned}
$$



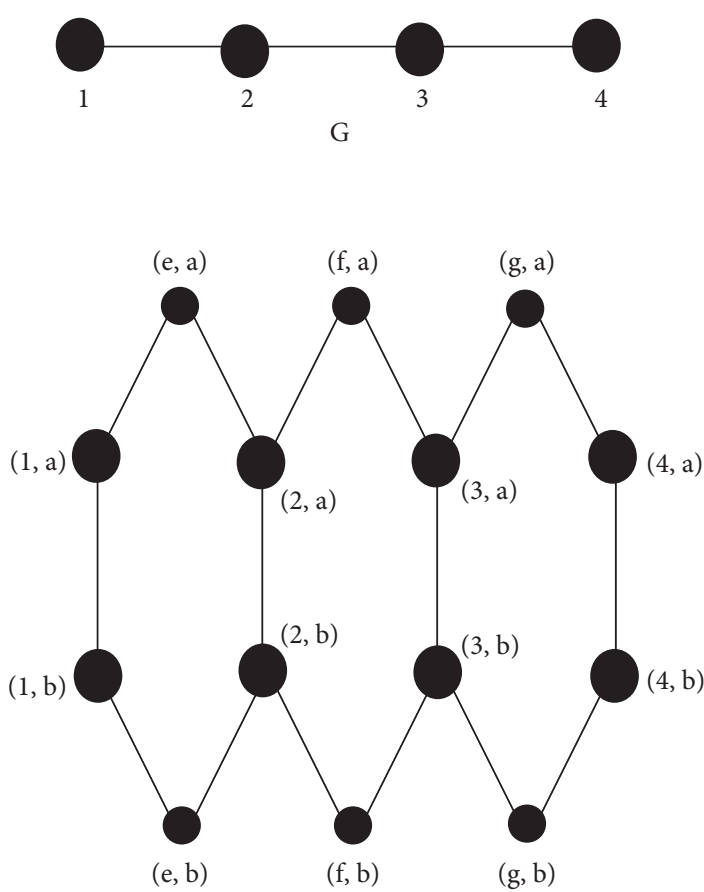

$\left(\mathrm{G}+{ }_{\mathrm{S}} \mathrm{H}\right)$

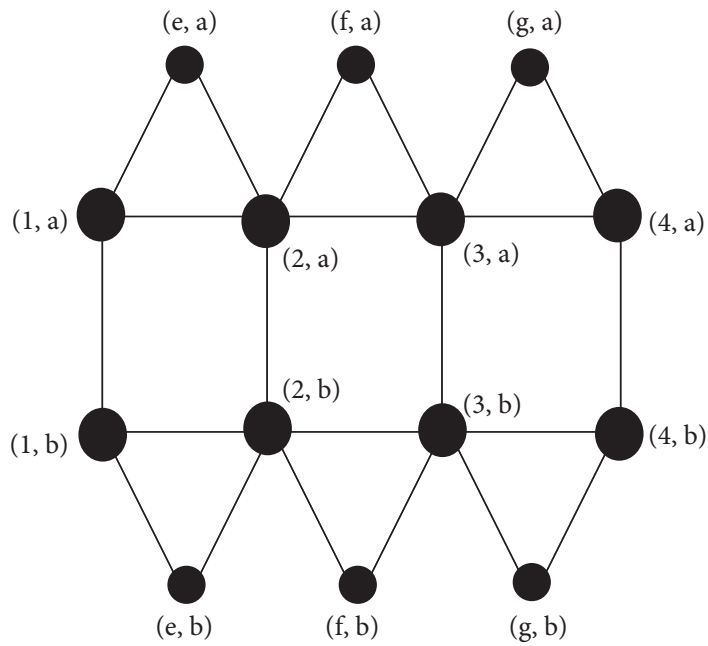

$\left(\mathrm{G}+{ }_{\mathrm{R}} \mathrm{H}\right)$

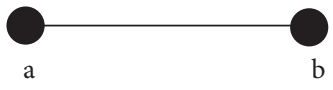

$\mathrm{H}$

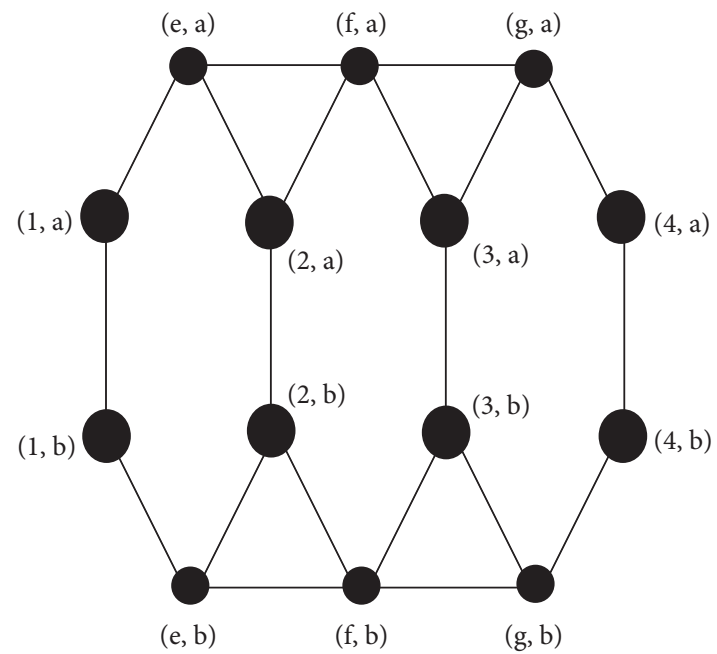

$\left(\mathrm{G}+{ }_{\mathrm{Q}} \mathrm{H}\right)$

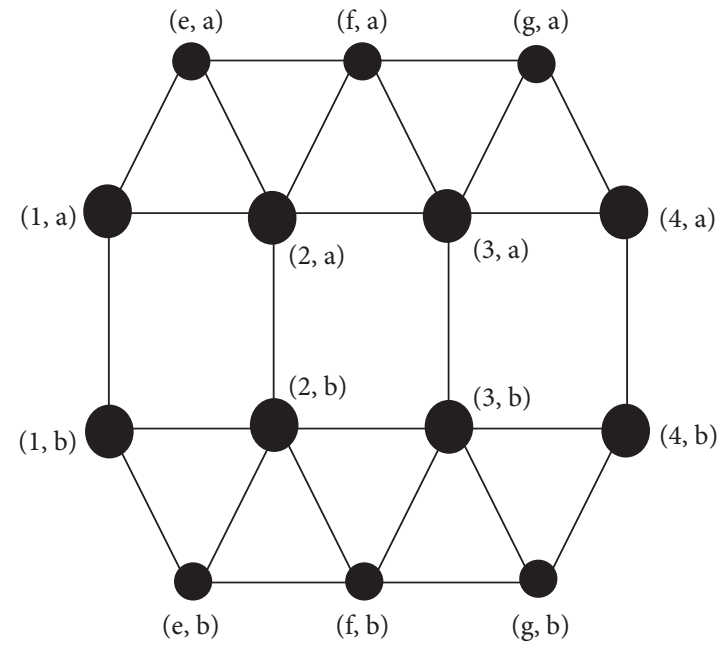

$\left(\mathrm{G}+{ }_{\mathrm{T}} \mathrm{H}\right)$

Figure 2 : Graphs $G \cong P_{4}, H \cong P_{2}$, and $G_{+F} H \cong P_{4+F} P_{2}$. 


$$
\begin{aligned}
& \sum B=\sum B_{1}+\sum B_{2}+\sum B_{3}+\sum B_{4}+\sum B_{5}+\sum B_{6} \\
& \sum B_{1}=\sum_{t \in V_{G_{1}}} \sum_{x_{1} x_{2} \notin E_{G_{2}}}\left[d\left(t, x_{1}\right)+d\left(t, x_{2}\right)\right] \\
& =\sum_{t \in V_{G_{1}}} \sum_{x_{1} x_{2} \notin E_{G_{2}}}\left[2 d_{G_{1}}(t)+d_{G_{2}}\left(x_{1}\right)+d_{G_{2}}\left(x_{2}\right)\right]=4 e_{1} \overline{e_{2}}+n_{1} \overline{M_{1}}\left(G_{2}\right), \\
& \sum B_{2}=\sum_{x \in V_{G_{2}}} \sum_{t_{1}, t_{2} \in V_{G_{1}}}\left[d\left(t_{1}, x\right)+d\left(t_{2}, x\right)\right] \\
& =\sum_{x \in V_{G_{2}}} \sum_{t_{1} t_{2} \in E_{G_{1}}}\left[d\left(t_{1}, x\right)+d\left(t_{2}, x\right)\right]+\sum_{x \in V_{G_{2}} t_{1} t_{2} \notin E_{G_{1}}}\left[d\left(t_{1}, x\right)+d\left(t_{2}, x\right)\right] \\
& =\sum_{x \in V_{G_{2}}} \sum_{t_{1} t_{2} \in E_{G_{1}}}\left[d_{G_{1}}\left(t_{1}\right)+d_{G_{1}}\left(t_{2}\right)+2 d_{G_{2}}(x)\right]+\sum_{x \in V_{G_{2}}} \sum_{t_{1} t_{2} \notin E_{G_{1}}}\left[d_{G_{1}}\left(t_{1}\right)+d_{G_{1}}\left(t_{2}\right)+2 d_{G_{2}}(x)\right] \\
& =M_{1}\left(G_{1}\right) n_{2}+4 e_{1} e_{2}+\overline{M_{1}}\left(G_{1}\right) n_{2}+4 \overline{e_{1}} e_{2}, \\
& \sum B_{3}=\sum_{t_{1} t_{2} \in E_{G_{1}}} \sum_{x_{1} x_{2} \in E_{G_{2}}}\left[d\left(t_{1}, x_{1}\right)+d\left(t_{2}, x_{2}\right)\right] \\
& =\sum_{t_{1} t_{2} \in E_{G_{1}}} \sum_{x_{1} x_{2} \in E_{G_{2}}}\left[d_{G_{1}}\left(t_{1}\right)+d_{G_{2}}\left(x_{1}\right)+d_{G_{1}}\left(t_{2}\right)+d_{G_{2}}\left(x_{2}\right)\right]=2\left[e_{2} M_{1}\left(G_{1}\right)+e_{1} M_{1}\left(G_{2}\right)\right] . \\
& \sum B_{4}=\sum_{t_{1} t_{2} \notin E_{G_{1}}} \sum_{x_{1} x_{2} \in E_{G_{2}}}\left[d\left(t_{1}, x_{1}\right)+d\left(t_{2}, x_{2}\right)\right] \\
& =\sum_{t_{1} t_{2} \notin E_{G_{1}}} \sum_{x_{1} x_{2} \in E_{G_{2}}}\left[d_{G_{1}}\left(t_{1}\right)+d_{G_{2}}\left(x_{1}\right)+d_{G_{1}}\left(t_{2}\right)+d_{G_{2}}\left(x_{2}\right)\right]=2\left[e_{2} \overline{M_{1}}\left(G_{1}\right)+\overline{e_{1}} M_{1}\left(G_{2}\right)\right], \\
& \sum B_{5}=\sum_{t_{1} t_{2} \notin E_{G_{1}}} \sum_{x_{1} x_{2} \notin E_{G_{2}}}\left[d\left(t_{1}, x_{1}\right)+d\left(t_{2}, x_{2}\right)\right] \\
& =\sum_{t_{1} t_{2} \notin E_{G_{1}}} \sum_{x_{1} x_{2} \notin E_{G_{2}}}\left[d_{G_{1}}\left(t_{1}\right)+d_{G_{2}}\left(x_{1}\right)+d_{G_{1}}\left(t_{2}\right)+d_{G_{2}}\left(x_{2}\right)\right]=2\left[\overline{e_{2}} \overline{M_{1}}\left(G_{1}\right)+\overline{e_{1}} \overline{M_{1}}\left(G_{2}\right)\right], \\
& \sum B_{6}=\sum_{t_{1} t_{2} \in E_{G_{1}}} \sum_{x_{1} x_{2} \notin E_{G_{2}}}\left[d\left(t_{1}, x_{1}\right)+d\left(t_{2}, x_{2}\right)\right] \\
& =\sum_{t_{1} t_{2} \in E_{G_{1}}} \sum_{x_{1} x_{2} \notin E_{G_{2}}}\left[d_{G_{1}}\left(t_{1}\right)+d_{G_{2}}\left(x_{1}\right)+d_{G_{1}}\left(t_{2}\right)+d_{G_{2}}\left(x_{2}\right)\right]=2\left[\bar{e}_{2} M_{1}\left(G_{1}\right)+e_{1} \overline{M_{1}}\left(G_{2}\right)\right], \\
& \sum B=4 e_{1} \bar{e}_{2}+e_{2}\left(e_{1}+\bar{e}_{1}\right)+\left(2\left(e_{2}+\bar{e}_{2}\right)+n_{2}\right) M_{1}\left(G_{1}\right)+\left(n_{2}\left(2 e_{2}+\bar{e}_{2}\right)\right) \bar{M}_{1}\left(G_{1}\right) \\
& +2\left(e_{2}+\bar{e}_{1}\right) M_{1}\left(G_{2}\right)+n_{1}+2\left(\bar{e}_{1}+e_{1}\right) \bar{M}_{1}\left(G_{2}\right) \text {, } \\
& \sum C=\sum C_{1}+\sum C_{2}+\sum C_{3} \\
& \sum C_{1}=\sum_{\substack{t_{1} t_{2} \notin E\left(S\left(G_{1}\right)\right) \\
\text { titeV } \\
t_{2} \in V\left(S\left(G_{1}\right)-V\left(G_{1}\right)\right)}} \sum_{\substack{x \in V_{G_{2}} \\
t_{1}}}\left[d\left(t_{1}, x\right)+d\left(t_{2}, x\right)\right], \\
& =\sum_{\substack{t_{1} t_{2} \notin E\left(S\left(G_{1}\right)\right) \\
t_{1} \in V\left(G_{1}\right) \\
t_{2} \in V\left(S\left(G_{1}\right)-V\left(G_{1}\right)\right)}} \sum_{\substack{t_{1} \\
G_{G_{2}}}}\left[d_{G_{1}}\left(t_{1}\right)+d(x)+d_{S\left(G_{1}\right)}\left(t_{2}\right)\right] \\
& =n_{2} \sum_{\substack{t_{1} t_{2} \notin E\left(S\left(G_{1}\right)\right) \\
\text { titeV(G) } \\
t_{2} \in V\left(S\left(G_{1}\right)-V\left(G_{1}\right)\right)}} d\left(t_{1}\right)+2 e_{1}\left(n_{1}-2\right)\left[e_{2}+n_{2}\right] .
\end{aligned}
$$




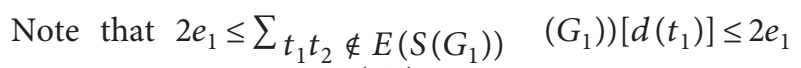
$\begin{array}{ll}\left(n_{1}-2\right) E\left(S\left(G_{1}\right)\right) . & t_{1} \in V\left(G_{1} \in V\left(S\left(G_{1}\right)-V\right.\right.\end{array}$

$$
\begin{aligned}
& 2 n_{2} e_{1}\left(n_{1}-2\right)+2 e_{2} e_{1}\left(n_{1}-2\right)+2 n_{2} e_{1}\left(n_{1}-2\right) \leq \sum C_{1} \leq 2 n_{2} e_{1}\left(n_{1}-2\right) E\left(S\left(G_{1}\right)\right)+2 e_{2} e_{1}\left(n_{1}-2\right)+2 n_{2} e_{1}\left(n_{1}-2\right), \\
& \sum C_{2}=\sum_{t_{1} t_{2} \notin E\left(S\left(G_{1}\right)\right)} \sum_{x_{1}, x_{2} \in V_{G_{2}}}\left[d\left(t_{1}, x_{1}\right)+d\left(t_{2}, x_{2}\right)\right] \\
& t_{1} \in V\left(G_{1}\right) \\
& t_{2} \in V\left(S\left(G_{1}\right)-V\left(G_{1}\right)\right) \\
& =\sum_{t_{1} t_{2} \notin E\left(S\left(G_{1}\right)\right)} \sum_{x_{1}, x_{2} \in V_{G_{2}}}\left[d_{G_{1}}\left(t_{1}\right)+d\left(x_{1}\right)+d_{S\left(G_{1}\right)}\left(t_{2}\right)\right] \\
& t_{1} \in V\left(G_{1}\right) \\
& t_{2} \in V\left(S\left(G_{1}\right)-V\left(G_{1}\right)\right) \\
& =n_{2}\left(n_{2}-1\right) \sum_{t_{1} t_{2} \notin E\left(S\left(G_{1}\right)\right)} \\
& t_{1} \in V\left(G_{1}\right) \\
& t_{2} \in V\left(S\left(G_{1}\right)-V\left(G_{1}\right)\right) \\
& \cdot\left[d_{G_{1}}\left(t_{1}\right)\right]+2 e_{2} e_{1}\left(n_{1}-2\right)\left(n_{2}-1\right)+2 n_{2}\left(n_{2}-1\right) e_{1}\left(n_{1}-2\right) \text {. }
\end{aligned}
$$

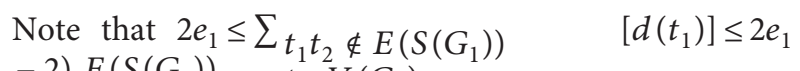

$$
\begin{array}{ll}
\left(n_{1}-2\right) E\left(S\left(G_{1}\right)\right) . \quad & t_{1} \in V\left(G_{1}\right) \\
& t_{2} \in V\left(S\left(G_{1}\right)-V\left(G_{1}\right)\right)
\end{array}
$$

$$
\begin{aligned}
& 2 n_{2}\left(n_{2}-1\right) e_{1}\left(n_{1}-2\right)+2 e_{2} e_{1}\left(n_{1}-2\right)\left(n_{2}-1\right)+2 n_{2}\left(n_{2}-1\right) e_{1}\left(n_{1}-2\right) \leq \sum C_{2} \leq 2 n_{2}\left(n_{2}-1\right) e_{1}\left(n_{1}-2\right) E\left(S\left(G_{1}\right)\right), \\
& +2 e_{2} e_{1}\left(n_{1}-2\right)\left(n_{2}-1\right)+2 n_{2}\left(n_{2}-1\right) e_{1}\left(n_{1}-2\right), \\
& \sum C_{3}=\sum_{t_{1} t_{2} \in E\left(S\left(G_{1}\right)\right)} \sum_{x_{1}, x_{2} \in V_{G_{2}}}\left[d\left(t_{1}, x_{1}\right)+d\left(t_{2}, x_{2}\right)\right]=\sum_{t_{1} t_{2} \in E\left(S\left(G_{1}\right)\right)} \sum_{x_{1}, x_{2} \in V_{G_{2}}}\left[d_{G_{1}}\left(t_{1}\right)+d\left(x_{1}\right)+d_{S\left(G_{1}\right)}\left(t_{2}\right)\right] \\
& t_{1} \in V\left(G_{1}\right) \\
& t_{2} \in V\left(S\left(G_{1}\right)-V *\left(G_{1}\right)\right) \quad t_{2} \in V\left(S\left(G_{1}\right)-V\left(G_{1}\right)\right) \\
& =n_{2}\left(n_{2}-1\right) \sum_{\substack{t_{1} t_{2} \in E\left(S\left(G_{1}\right)\right) \\
t_{1} \in V\left(G_{1}\right) t_{2} \in V\left(S\left(G_{1}\right)-V\left(G_{1}\right)\right)}}\left[d\left(t_{1}\right)\right]+2 e_{2}\left(n_{2}-1\right)\left|\sum_{\substack{t_{1} t_{2} \in E\left(S\left(G_{1}\right)\right) \\
t_{1} \in V\left(G_{1}\right) t_{2} \in V\left(S\left(G_{1}\right)-V\left(G_{1}\right)\right)}} d\left(t_{1}\right)\right|+2 n_{2}\left(n_{2}-1\right) \mid \\
& \sum_{\substack{t_{1} t_{2} \in E\left(S\left(G_{1}\right)\right) \\
t_{1} \in V\left(G_{1}\right) t_{2} \in V}}\left(S\left(G_{1}\right)-G_{1}\right) d\left(t_{1}\right) \mid
\end{aligned}
$$


Note that $2 e_{1} \leq \sum_{t_{1} t_{2} \in E\left(S\left(G_{1}\right)\right)}$

$\left[d\left(t_{1}\right)\right] \leq 2 e_{1} E\left(S\left(G_{1}\right)\right)$.

$$
t_{1} \in V\left(G_{1}\right) t_{2} \in V\left(S\left(G_{1}\right)-V\left(G_{1}\right)\right)
$$

$$
2 e_{1} n_{2}\left(n_{2}-1\right)+4 e_{1}\left(n_{2}-1\right)\left(e_{2}+n_{2}\right) \leq \sum C_{3} \leq 2 e_{1} n_{2}\left(n_{2}-1\right) E\left(S\left(G_{1}\right)\right)+4 e_{1}\left(n_{2}-1\right)\left(e_{2}+n_{2}\right) .
$$

Consequently,

$$
\begin{aligned}
& 2 e_{1} n_{2}\left(n_{2}-1\right)+4 e_{1}\left(n_{2}-1\right)\left(e_{2}+n_{2}\right)+2 n_{2}\left(n_{2}-1\right) e_{1}\left(n_{1}-2\right)+ \\
& 2 e_{2} e_{1}\left(n_{1}-2\right)\left(n_{2}-1\right)+2 n_{2}\left(n_{2}-1\right) e_{1}\left(n_{1}-2\right)+2 n_{2} e_{1}\left(n_{1}-2\right)+2 e_{2} e_{1}\left(n_{1}-2\right)+2 n_{2} e_{1}\left(n_{1}-2\right) \\
& \leq \\
& \sum C \leq \\
& 2 e_{1} n_{2}\left(n_{2}-1\right) E\left(S\left(G_{1}\right)\right)+4 e_{1}\left(n_{2}-1\right)\left(e_{2}+n_{2}\right)+2 n_{2}\left(n_{2}-1\right) e_{1}\left(n_{1}-2\right) E\left(S\left(G_{1}\right)\right)+ \\
& 2 e_{2} e_{1}\left(n_{1}-2\right)\left(n_{2}-1\right)+2 n_{2}\left(n_{2}-1\right) e_{1}\left(n_{1}-2\right)+n_{2} e_{1}\left(n_{1}-2\right) E\left(S\left(G_{1}\right)\right)+2 e_{2} e_{1}\left(n_{1}-2\right)+2 n_{2} e_{1}\left(n_{1}-2\right) . \\
& \text { By putting the values of } \sum A+\sum B+\sum C \text { in equation (5), } \quad \alpha_{1} \leq \bar{M}\left(G_{1+R} G_{2}\right) \leq \alpha_{2},
\end{aligned}
$$
we get the required proof.

where

Theorem 2. Let $G_{1}$ and $G_{2}$ be two connected graphs; then, first Zagreb coindex of $G_{1+R} G_{2}$ is given as

$$
\begin{aligned}
\alpha_{1}= & 2\left(n_{2}^{2} e_{1}^{2}-n_{2} e_{1}\right)+4\left(4 e_{1} \bar{e}_{2}+e_{2} \bar{e}_{1}\right)+2 e_{1} n_{2}\left(n_{1}-2\right)\left(e_{2}+n_{2}\right)+\left(4 e_{2}+4 \bar{e}_{2}\right) M_{1}\left(G_{1}\right)+2\left(e_{1}+\bar{e}_{1}\right) M_{1}\left(G_{2}\right) \\
& +2\left(n_{2}+2\left(e_{2}+\bar{e}_{2}\right)\right) \bar{M}_{1}\left(G_{1}\right)+\left(n_{1}+2 \bar{e}_{1}+2 e_{1}\right) \bar{M}_{1}\left(G_{2}\right)+2 e_{1}\left[n_{2}^{2}\left(2 n_{2}-1\right)+2\left(n_{2}-1\right)\left(e_{2}+n_{2}\right)\right], \\
\alpha_{2}= & 2\left(n_{2}^{2} e_{1}^{2}-n_{2} e_{1}\right)+4\left(4 e_{1} \bar{e}_{2}+e_{2} \bar{e}_{1}\right)+2 e_{1} n_{2}\left(n_{1}-2\right)\left(e_{2}+n_{2}\right)+\left(4 e_{2}+4 \bar{e}_{2}\right) M_{1}\left(G_{1}\right)+2\left(n_{2}+2\left(e_{2}+\bar{e}_{2}\right)\right) \bar{M}_{1}\left(G_{1}\right) \\
& +2\left(e_{1}+\bar{e}_{1}\right) M_{1}\left(G_{2}\right)+\left(n_{1}+2 \bar{e}_{1}+2 e_{1}\right) \bar{M}_{1}\left(G_{2}\right)+2 E\left(R\left(G_{1}\right)\right) e_{1}\left[n_{2}^{2}\left(2 n_{2}-1\right)\left(n_{1}-2\right)+2\left(n_{2}-1\right)\left(e_{2}+n_{2}\right)\right] .
\end{aligned}
$$

Proof. Using equation (2), we have

$$
\begin{aligned}
& \bar{M}\left(G_{1+R} G_{2}\right)=\sum_{\left(t_{1}, t_{2}\right)\left(x_{1}, x_{2}\right) \notin E\left(G_{1+R} G_{2}\right)}\left[d\left(t_{1}, x_{1}\right)+d\left(t_{2}, x_{2}\right)\right], \\
& \bar{M}\left(G_{1+R} G_{2}\right)=\sum A+\sum B+\sum C .
\end{aligned}
$$


Using equation (6), we directly have

$$
\begin{aligned}
& \sum A=2\left(n_{2}^{2} e_{1}^{2}-n_{2} e_{1}\right) \\
& \sum B=\sum B_{1}+\sum B_{2}+\sum B_{3}+\sum B_{4}+\sum B_{5}+\sum B_{6} \\
& \sum B_{1}=\sum_{t \in V_{G_{1}}} \sum_{x_{1} x_{2} \notin E_{G_{2}}}\left[d\left(t, x_{1}\right)+d\left(t, x_{2}\right)\right] \\
& =\sum_{t \in V_{G_{1}}} \sum_{x_{1} x_{2} \notin E_{G_{2}}}\left[4 d_{\left(G_{1}\right)}(t)+d_{G_{2}}\left(x_{1}\right)+d_{G_{2}}\left(x_{2}\right)\right]=8 e_{1} \overline{e_{2}}+n_{1} \overline{M_{1}}\left(G_{2}\right), \\
& \sum B_{2}=\sum_{x \in V_{G_{2}}} \sum_{t_{1}, t_{2} \in V_{G_{1}}}\left[d\left(t_{1}, x\right)+d\left(t_{2}, x\right)\right] \\
& =\sum_{x \in V_{G_{2}} t_{1} t_{2} \notin E_{G_{1}}}\left[d\left(t_{1}, x\right)+d\left(t_{2}, x\right)\right]=\sum_{x \in V_{G_{2}}\left(t_{1} t_{2}\right) \notin E_{G_{1}}}\left[d_{R\left(G_{1}\right)}\left(t_{1}\right)+d_{R\left(G_{1}\right)}\left(t_{2}\right)+2 d_{G_{2}}(x)\right] \\
& =\sum_{x \in V_{G_{2}}\left(t_{1} t_{2}\right) \notin E_{G_{1}}}\left[2 d_{\left(G_{1}\right)}\left(t_{1}\right)+2 d_{\left(G_{1}\right)}\left(t_{2}\right)+2 d_{G_{2}}(x)\right]=\left[2 \overline{M_{1}}\left(G_{1}\right) n_{2}+4 \overline{e_{1}} e_{2}\right], \\
& \sum B_{3}=\sum_{t_{1} t_{2} \in E_{G_{1}}} \sum_{x_{1} x_{2} \in E_{G_{2}}}\left[d\left(t_{1}, x_{1}\right)+d\left(t_{2}, x_{2}\right)\right] \\
& =2\left[\sum_{t_{1} t_{2} \in E_{G_{1}}} \sum_{x_{1} x_{2} \in E_{G_{2}}}\left[d_{R\left(G_{1}\right)}\left(t_{1}\right)+d_{G_{2}}\left(x_{1}\right)+d_{R\left(G_{1}\right)}\left(t_{2}\right)+d_{G_{2}}\left(x_{2}\right)\right]\right. \\
& =2\left[\sum_{t_{1} t_{2} \in E_{G_{1}}} \sum_{x_{1} x_{2} \in E_{G_{2}}}\left[2 d_{\left(G_{1}\right)}\left(t_{1}\right)+d_{G_{2}}\left(x_{1}\right)+2 d_{\left(G_{1}\right)}\left(t_{2}\right)+d_{G_{2}}\left(x_{2}\right)\right]=2\left[2 e_{2} M_{1}\left(G_{1}\right)+e_{1} M_{1}\left(G_{2}\right)\right],\right. \\
& \sum B_{4}=\sum_{t_{1} t_{2} \notin E_{G_{1}}} \sum_{x_{1} x_{2} \in E_{G_{2}}}\left[d\left(t_{1}, x_{1}\right)+d\left(t_{2}, x_{2}\right)\right] \\
& =2\left[\sum_{t_{1} t_{2} \notin E_{G_{1}}} \sum_{x_{1} x_{2} \in E_{G_{2}}}\left[d_{R\left(G_{1}\right)}\left(t_{1}\right)+d_{G_{2}}\left(x_{1}\right)+d_{R\left(G_{1}\right)}\left(t_{2}\right)+d_{G_{2}}\left(x_{2}\right)\right]\right. \\
& =2\left[\sum_{t_{1} t_{2} \notin E_{G_{1}}} \sum_{x_{1} x_{2} \in E_{G_{2}}}\left[2 d_{\left(G_{1}\right)}\left(t_{1}\right)+d_{G_{2}}\left(x_{1}\right)+2 d_{\left(G_{1}\right)}\left(t_{2}\right)+d_{G_{2}}\left(x_{2}\right)\right]=2\left[2 e_{2} \overline{M_{1}}\left(G_{1}\right)+\overline{e_{1}} M_{1}\left(G_{2}\right)\right],\right. \\
& \sum B_{5}=\sum_{t_{1} t_{2} \notin E_{G_{1}}} \sum_{x_{1} x_{2} \notin E_{G_{2}}}\left[d\left(t_{1}, x_{1}\right)+d\left(t_{2}, x_{2}\right)\right] \\
& =2\left[\sum_{t_{1} t_{2} \notin E_{G_{1}}} \sum_{x_{1} x_{2} \notin E_{G_{2}}}\left[d_{R\left(G_{1}\right)}\left(t_{1}\right)+d_{G_{2}}\left(x_{1}\right)+d_{R\left(G_{1}\right)}\left(t_{2}\right)+d_{G_{2}}\left(x_{2}\right)\right]\right] \\
& =2\left[\sum_{t_{1} t_{2} \notin E_{G_{1}}} \sum_{x_{1} x_{2} \notin E_{G_{2}}}\left[d_{\left(G_{1}\right)}\left(t_{1}\right)+d_{G_{2}}\left(x_{1}\right)+d_{\left(G_{1}\right)}\left(t_{2}\right)+d_{G_{2}}\left(x_{2}\right)\right]=2\left[2 \overline{e_{2}} \overline{M_{1}}\left(G_{1}\right)+\overline{e_{1}} \overline{M_{1}}\left(G_{2}\right)\right],\right. \\
& \sum B_{6}=\sum_{t_{1} t_{2} \in E_{G_{1}}} \sum_{x_{1} x_{2} \notin E_{G_{2}}}\left[d\left(t_{1}, x_{1}\right)+d\left(t_{2}, x_{2}\right)\right] \\
& =2\left[\sum_{t_{1} t_{2} \in E_{G_{1}}} \sum_{x_{1} x_{2} \notin E_{G_{2}}}\left[d_{R\left(G_{1}\right)}\left(t_{1}\right)+d_{G_{2}}\left(x_{1}\right)+d_{R\left(G_{1}\right)}\left(t_{2}\right)+d_{G_{2}}\left(x_{2}\right)\right]\right. \\
& =2\left[\sum_{t_{1} t_{2} \in E_{G_{1}}} \sum_{x_{1} x_{2} \notin E_{G_{2}}}\left[2 d_{\left(G_{1}\right)}\left(t_{1}\right)+d_{G_{2}}\left(x_{1}\right)+2 d_{\left(G_{1}\right)}\left(t_{2}\right)+d_{G_{2}}\left(x_{2}\right)\right]=2\left[2 \bar{e}_{2} M_{1}\left(G_{1}\right)+e_{1} \bar{M}_{1}\left(G_{2}\right)\right]\right. \text {, }
\end{aligned}
$$




$$
\begin{aligned}
& \sum B=4\left(2 e_{1} \bar{e}_{2}+4 \bar{e}+e_{2} \bar{e}_{1}\right)+\left(4 e_{2}+4 \bar{e}_{2}\right) M_{1}\left(G_{1}\right)+2\left(e_{1}+\bar{e}_{1}\right) M_{1}\left(G_{2}\right) \\
& +2 n_{2}+2\left(e_{2}+\bar{e}_{2}\right) \bar{M}_{1}\left(G_{1}\right)+\left(n_{1}+2 \bar{e}_{1}+2 e_{1}\right) \bar{M}_{1}\left(G_{2}\right) \\
& \sum C=\sum_{\substack{t_{1}, t_{2} \in V\left(R\left(G_{1}\right)\right) \\
t_{1} \in V\left(G_{1}\right) \\
t_{2} \in V\left(R\left(G_{1}\right)-V\left(G_{1}\right)\right)}}\left[d\left(t_{1}, x_{1}\right)+d\left(t_{2}, x_{2}\right)\right] \\
& =\sum_{\substack{t_{1} t_{2} \notin E\left(R\left(G_{1}\right)\right) \\
t_{1} \in V\left(G_{1}\right) \\
t_{2} \in V\left(R\left(G_{1}\right)-V\left(G_{1}\right)\right)}} \sum_{x \in G_{G_{2}}}\left[d\left(t_{1}, x\right)+d\left(t_{2}, x\right)\right]+\sum_{\substack{t_{1} t_{2} \notin E\left(R\left(G_{1}\right)\right) \\
t_{1} \in V\left(G_{1}\right) \\
t_{2} \in V\left(R G_{1}-V\left(G_{1}\right)\right)}} \sum_{x_{1}, x_{2} \in V_{G_{2}}}\left[d\left(t_{1}, x_{1}\right)+d\left(t_{2}, x_{2}\right)\right]
\end{aligned}
$$

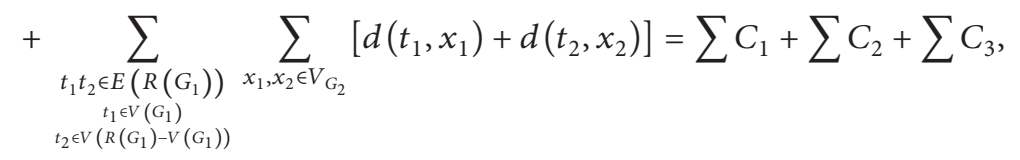

$$
\begin{aligned}
& \sum C_{1}=\sum_{\substack{t_{1} t_{2} \notin E\left(R\left(G_{1}\right)\right) \\
\text { ant } \\
t_{2} \in V\left(G_{1}\right)}} \sum_{\left.\left.x \in V_{G_{2}}\right)-V\left(G_{1}\right)\right)}\left[d\left(t_{1}, x\right)+d\left(t_{2}, x\right)\right] \\
& =\sum_{\substack{t_{1} t_{2} \notin E\left(R\left(G_{1}\right)\right) \\
t_{1} \in V\left(G_{1}\right) \\
t_{2} \in V\left(R\left(G_{1}\right)-V\left(G_{1}\right)\right)}} \sum_{x \in V_{G_{2}}}\left[d_{R\left(G_{1}\right)}\left(t_{1}\right)+d(x)+d_{R\left(G_{1}\right)}\left(t_{2}\right)\right]=\sum_{\substack{t_{1} t_{2} \notin E\left(R\left(G_{1}\right)\right) \\
\text { titeV(G) } \\
t_{2} \in V\left(R\left(G_{1}\right)-V\left(G_{1}\right)\right)}} \sum_{x \in V_{G_{2}}}\left[d_{R\left(G_{1}\right)}\left(t_{1}\right)+d(x)+2\right] \\
& =n_{2} \sum_{\substack{t_{1} t_{2} \notin E\left(R\left(G_{1}\right)\right) \\
t_{1} \in V\left(G_{1}\right) \\
t_{2} \in V\left(R\left(G_{1}\right)-V\left(G_{1}\right)\right)}} d_{R G_{1}}\left(t_{1}\right)+2 e_{2} e_{1}\left(n_{2}-1\right)+2 n_{2} e_{1}\left(n_{2}-1\right) .
\end{aligned}
$$


Note that $2 e_{1} \leq \sum_{t_{1} t_{2} \notin E\left(R\left(G_{1}\right)\right) t_{1} \in V\left(G_{1}\right) t_{2} \in V\left(S\left(G_{1}\right)-V\left(G_{1}\right)\right)}$ $\left[d\left(t_{1}\right)\right] \leq 2 E\left(R\left(G_{1}\right)\right) e_{1}\left(n_{1}-2\right)$.

$$
\begin{aligned}
& 2 n_{2} e_{1}+2 e_{2} e_{1}\left(n_{2}-1\right)+2 n_{2} e_{1}\left(n_{2}-1\right) \leq \sum C_{1} \leq 2 n_{2} E\left(R\left(G_{1}\right)\right) e_{1}\left(n_{1}-2\right)+2 e_{2} e_{1}\left(n_{2}-1\right)+2 n_{2} e_{1}\left(n_{2}-1\right) \\
& \sum C_{2}=\sum_{t_{1} t_{2} \notin E\left(R\left(G_{1}\right)\right)} \sum_{\left(x_{1}, x_{2}\right) \in V_{G_{2}}}\left[d\left(t_{1}, x_{1}\right)+d\left(t_{2}, x_{2}\right)\right] \\
& t_{1} \in V\left(G_{1}\right) \\
& t_{2} \in V\left(R\left(G_{1}\right)-V\left(G_{1}\right)\right) \\
& =\sum_{t_{1} t_{2} \notin E\left(R\left(G_{1}\right)\right)} \sum_{x_{1}, x_{2} \in V_{G_{2}}}\left[d_{R\left(G_{1}\right)}\left(t_{1}\right)+d\left(x_{1}\right)+d_{S\left(G_{1}\right)}\left(t_{2}\right)\right] \\
& t_{1} \in V\left(G_{1}\right) \\
& t_{2} \in V\left(R\left(G_{1}\right)-V\left(G_{1}\right)\right) \\
& =\sum_{t_{1} t_{2} \notin E\left(R\left(G_{1}\right)\right)} \sum_{x_{1}, x_{2} \in V_{G_{2}}}\left[d_{R\left(G_{1}\right)}\left(t_{1}\right)+d\left(x_{1}\right)+2\right] \\
& t_{1} \in V\left(G_{1}\right) \\
& t_{2} \in V\left(R\left(G_{1}\right)-V\left(G_{1}\right)\right) \\
& =n_{2}\left(n_{2}-1\right) \sum_{\substack{t_{1} t_{2} \notin E\left(R\left(G_{1}\right)\right) \\
t_{1} \in V\left(G_{1}\right) t_{2} \in V\left(R\left(G_{1}\right)-V\left(G_{1}\right)\right)}} d_{R G_{1}}\left(t_{1}\right)+2 e_{2} e_{1}\left(n_{1}-2\right)\left(n_{2}-1\right) \\
& +2 n_{2}\left(n_{2}-1\right) e_{1}\left(n_{1}-2\right) \text {. }
\end{aligned}
$$

Note that $2 e_{1} \leq \sum_{t_{1} t_{2} \notin E\left(R\left(G_{1}\right)\right) t_{1} \in V\left(G_{1}\right) t_{2} \in V\left(S\left(G_{1}\right)-V\left(G_{1}\right)\right)}$ $\left[d\left(t_{1}\right)\right] \leq 2 E\left(R\left(G_{1}\right)\right) e_{1}\left(n_{1}-2\right)$.

$$
\begin{aligned}
& 2 n_{2}\left(n_{2}-1\right) e_{1}\left(n_{1}-2\right)+2 e_{2} e_{1}\left(n_{1}-2\right)\left(n_{2}-1\right)+2 n_{2}\left(n_{2}-1\right) e_{1}\left(n_{1}-2\right) \\
& \leq \sum C_{2} \leq 2 n_{2}\left(n_{2}-1\right) E\left(R\left(G_{1}\right)\right) e_{1}\left(n_{1}-2\right) \\
& +2 e_{2} e_{1}\left(n_{1}-2\right)\left(n_{2}-1\right)+2 n_{2}\left(n_{2}-1\right) e_{1}\left(n_{1}-2\right), \\
& \sum C_{3}=\sum_{t_{1} t_{2} \in E\left(R\left(G_{1}\right)\right)} \sum_{x_{1}, x_{2} \in V_{G_{2}}}\left[d\left(t_{1}, x_{1}\right)+d\left(t_{2}, x_{2}\right)\right] \\
& t_{1} \in V\left(G_{1}\right) \\
& t_{2} \in V\left(R\left(G_{1}\right)-V\left(G_{1}\right)\right) \\
& \begin{array}{c}
=\sum_{\substack{t_{1} t_{2} \in E\left(R\left(G_{1}\right)\right) \\
t_{1} \in V\left(G_{1}\right)}} \sum_{\left(x_{1}, x_{2}\right) \in V_{G_{2}}}\left[d_{R\left(G_{1}\right)}\left(t_{1}\right)+d\left(x_{1}\right)+d_{R\left(G_{1}\right)}\left(t_{2}\right)\right]+\sum_{\substack{t_{1} t_{2} \in E\left(R\left(G_{1}\right)\right) \\
t_{1} \in V\left(G_{1}\right)}}\left[d_{R\left(G_{1}\right)}\left(t_{1}\right)+d\left(x_{1}\right)+2\right] \\
t_{2} \in V\left(R\left(G_{1}\right)-V\left(G_{1}\right)\right)
\end{array} \\
& =n_{2}\left(n_{2}-1\right) \sum_{\substack{t_{1} t_{2} \in E\left(R\left(G_{1}\right)\right) \\
t_{1} \in V\left(G_{1}\right) t_{2} \in V\left(R\left(G_{1}\right)-V\left(G_{1}\right)\right)}} d_{R\left(G_{1}\right)}\left(t_{1}\right)+2 e_{2}\left(n_{2}-1\right)\left|\sum_{\substack{t_{1} t_{2} \in E\left(R\left(G_{1}\right)\right) \\
t_{1} \in V\left(G_{1}\right) t_{2} \in V\left(R\left(G_{1}\right)-V\left(G_{1}\right)\right)}} d_{R\left(G_{1}\right)}\left(t_{1}\right)\right| \\
& +2 n_{2}\left(n_{2}-1\right)\left|\sum_{\substack{t_{1} t_{2} \in E\left(R\left(G_{1}\right)\right) \\
t_{1} \in V\left(G_{1}\right) t_{2} \in V\left(R\left(G_{1}\right)-V\left(G_{1}\right)\right)}} d_{R\left(G_{1}\right)}\left(t_{1}\right)\right| .
\end{aligned}
$$


Note that $2 e_{1} \leq \sum_{t_{1} t_{2} \in E\left(R\left(G_{1}\right)\right)}$

$d\left(t_{1}\right) \leq 4 E\left(R\left(G_{1}\right)\right) e_{1}$. So,

$t_{1} \in V\left(G_{1}\right) t_{2} \in V\left(S\left(G_{1}\right)-V\left(G_{1}\right)\right)$

$$
4 e_{1}\left[n_{2}\left(n_{2}-1\right)+2 e_{2}\left(n_{2}-1\right)+2 n_{2}\left(n_{2}-1\right)\right] \leq \sum C_{3} \leq 4 e_{1} E\left(R\left(G_{1}\right)\right)\left[n_{2}\left(n_{2}-1\right)+2 e_{2}\left(n_{2}-1\right)+2 n_{2}\left(n_{2}-1\right)\right] .
$$

Consequently,

$$
\begin{aligned}
2 n_{2} e_{1} & +2 e_{2} e_{1}\left(n_{2}-1\right)+2 n_{2} e_{1}\left(n_{2}-1\right)+2 n_{2}\left(n_{2}-1\right) e_{1}\left(n_{1}-2\right)+2 e_{2} e_{1}\left(n_{1}-2\right)\left(n_{2}-1\right) \\
& +2 n_{2}\left(n_{2}-1\right) e_{1}\left(n_{1}-2\right)+4 e_{1}\left[n_{2}\left(n_{2}-1\right)+2 e_{2}\left(n_{2}-1\right)+2 n_{2}\left(n_{2}-1\right)\right] \\
\leq & \sum C \leq \\
& 2 n_{2} E\left(R\left(G_{1}\right)\right) e_{1}\left(n_{1}-2\right)+2 e_{2} e_{1}\left(n_{2}-1\right)+2 n_{2} e_{1}\left(n_{2}-1\right)+2 n_{2}\left(n_{2}-1\right) E\left(R\left(G_{1}\right)\right) e_{1}\left(n_{1}-2\right)+2 e_{2} e_{1}\left(n_{1}-2\right)\left(n_{2}-1\right) \\
& +2 n_{2}\left(n_{2}-1\right) e_{1}\left(n_{1}-2\right)+4 e_{1} E\left(R\left(G_{1}\right)\right)\left[n_{2}\left(n_{2}-1\right)+2 e_{2}\left(n_{2}-1\right)+2 n_{2}\left(n_{2}-1\right)\right]
\end{aligned}
$$

$$
\alpha_{1} \leq \bar{M}\left(G_{1+Q} G_{2}\right) \leq \alpha_{2}
$$

By putting the values of $\sum A+\sum B+\sum C$ in equation where (16), we get the required proof.

Theorem 3. Let $G_{1}$ and $G_{2}$ be two connected graphs; then, first Zagreb coindex of $G_{1+Q} G_{2}$ is given as

$$
\begin{aligned}
\alpha_{1}= & 4\left(e_{1} \bar{e}_{2}+e_{2}\left(e_{1}+\bar{e}_{1}\right)\right)+2\left(n_{2}-1+\bar{e}_{2}\right)\left(2 e_{1}+\bar{e}_{1}\right)+2 e_{1}\left(n_{1}-2\right)\left(e_{2}+3 n_{2}\right)+n_{2}\left(2 n_{2}-1\right) e_{1} \\
& +2\left(2 e_{2}+3 n_{2}\right)\left(n_{2}-1\right) e_{1}+\left(n_{2}+2 e_{2}+2 \bar{e}_{2}\right) M_{1}\left(G_{1}\right)+2\left(e_{2}+\bar{e}_{2}\right) \\
& \bar{M}_{1}\left(G_{1}\right)+2\left(e_{1}+\bar{e}_{1}\right) M_{1}\left(G_{2}\right)+\left(n_{1}+2 \bar{e}_{1}+2 e_{1}\right) \bar{M}_{1}\left(G_{2}\right), \\
\alpha_{2}= & 4\left(e_{1} \bar{e}_{2}+e_{2}\left(e_{1}+\bar{e}_{1}\right)\right)+e_{1}\left(n_{1}-2\right)\left(2 e_{2}+3 n_{2}\right)+2 e_{1}\left(2 e_{2}+3 n_{2}\right)\left(n_{2}-1\right)+\left(n_{2}+2 e_{2}+2 \bar{e}_{2}\right) M_{1}\left(G_{1}\right) \\
& +\left(n_{2}+2\left(e_{2}+\bar{e}_{2}\right)\right) \bar{M}_{1}\left(G_{1}\right)+2\left(e_{1}+\bar{e}_{1}\right) M_{1}\left(G_{2}\right)+\left(n_{1}+2 \bar{e}_{1}+2 e_{1}\right) \bar{M}_{1}\left(G_{2}\right) \\
& +n_{2} \bar{M}_{1}\left(Q\left(G_{1}\right)\right)+4\left(\left(n_{2}-1\right)+\overline{e_{2}}\right) E\left(Q\left(G_{1}\right)\right) \\
& +2 n_{2}^{2} E\left(Q\left(G_{1}\right)\right) e_{1}\left(n_{1}-2\right)+2\left(n_{2}-1+\bar{e}_{2}\right) M_{1}\left(Q\left(G_{1}\right)\right) \bar{M}_{1}\left(Q\left(G_{1}\right)\right)+4 n_{2} e_{1} E\left(Q=\left(G_{1}\right)\right)\left(n_{2}-1\right) .
\end{aligned}
$$

Proof. Using equation (2), we have

$$
\begin{aligned}
& \bar{M}\left(G_{1+Q} G_{2}\right)=\sum_{\left(t_{1}, t_{2}\right)\left(x_{1}, x_{2}\right) \notin E\left(G_{1+Q} G_{2}\right)}\left[d\left(t_{1}, x_{1}\right)+d\left(t_{2}, x_{2}\right)\right], \\
& \bar{M}\left(G_{1+Q} G_{2}\right)= \sum A+\sum B+\sum C, \\
& \sum A= \sum A_{1}+\sum A_{2}+\sum A_{3}+\sum A_{4}+\sum A_{5}+\sum A_{6}+\sum A_{7}, \\
& \sum A_{1}=\sum_{\substack{t_{1} t_{2} \notin E\left(Q\left(G_{1}\right)\right) \\
t_{1}, t_{2} \in V\left(Q\left(G_{1}\right)-V\left(G_{1}\right)\right)}} \sum_{x \in V_{G_{2}}}\left[d\left(t_{1}, x\right)+d\left(t_{2}, x\right)\right]=n_{2} \sum_{\substack{t_{1} t_{2} \notin E\left(Q\left(G_{1}\right)\right) \\
t_{1}, t_{2} \in V\left(Q\left(G_{1}\right)-V\left(G_{1}\right)\right)}}\left[d_{Q G_{1}}\left(t_{1}\right)+d_{Q G_{1}}\left(t_{2}\right)\right] .
\end{aligned}
$$


Note that $0 \leq \sum_{t_{1} t_{2} \notin E\left(Q\left(G_{1}\right)\right)}$

$\left[d_{\mathrm{QG}_{1}}\left(t_{1}\right)+d_{\mathrm{QG}_{1}}\left(t_{2}\right)\right] \leq \bar{M}_{1}\left(\mathrm{Q}\left(G_{1}\right)\right)$, so

$$
t_{1}, t_{2} \in V\left(Q\left(G_{1}\right)-V\left(G_{1}\right)\right)
$$

$$
\begin{aligned}
0 & \leq \sum A_{1} \leq n_{2} \bar{M}_{1}\left(Q\left(G_{1}\right)\right) \\
\sum A_{2} & =\sum_{t \in V\left(Q\left(G_{1}\right)-V\left(G_{1}\right)\right)} \sum_{x_{1} x_{2} \in E_{G_{2}}}\left[d\left(t, x_{1}\right)+d\left(t, x_{2}\right)\right] \\
& =\sum_{t \in V\left(Q\left(G_{1}\right)-V\left(G_{1}\right)\right)} \sum_{x_{1} x_{2} \in E_{G_{2}}}\left[d_{Q G_{1}}(t)+d_{Q G_{1}}(t)\right]=2\left(n_{2}-1\right) \sum_{t \in V\left(Q\left(G_{1}\right)-\left(G_{1}\right)\right)}\left[d_{Q G_{1}}(t)\right] .
\end{aligned}
$$

so

Note that $e_{1} \leq \sum_{t \in V\left(Q\left(G_{1}\right)-V\left(G_{1}\right)\right)}\left[d_{Q_{G_{1}}}(t)\right] \leq 2 E\left(Q\left(G_{1}\right)\right)$,

$$
\begin{aligned}
2\left(n_{2}-1\right) e_{1} & \leq \sum A_{2} \leq 4\left(n_{2}-1\right) E\left(Q\left(G_{1}\right)\right), \\
\sum A_{3} & =\sum_{t \in V\left(Q\left(G_{1}\right)-V\left(G_{1}\right)\right)} \sum_{x_{1} x_{2} \notin E_{G_{2}}}\left[d\left(t, x_{1}\right)+d\left(t, x_{2}\right)\right] \\
& =\sum_{t \in V\left(Q\left(G_{1}\right)-V\left(G_{1}\right)\right)} \sum_{x_{1} x_{2} \notin E_{G_{2}}}\left[d_{Q G_{1}}(t)+d_{Q G_{1}}(u)\right]=2 \overline{e_{2}} \sum_{t \in V\left(Q\left(G_{1}\right)-\left(G_{1}\right)\right)}\left[d_{Q G_{1}}(u)\right] .
\end{aligned}
$$

Note that $e_{1} \leq \sum_{t \in V\left(Q\left(G_{1}\right)-V\left(G_{1}\right)\right)}\left[d_{\mathrm{QG}_{1}}(t)\right] \leq 2 E\left(Q\left(G_{1}\right)\right)$,

so

$$
\begin{aligned}
2 \overline{e_{2}} e_{1} & \leq \sum A_{3} \leq 4 \overline{e_{2}} E\left(Q\left(G_{1}\right)\right), \\
\sum A_{4} & \sum_{\substack{t_{1} t_{2} \in E\left(Q\left(G_{1}\right)\right) \\
t_{1}, t_{2} \in V\left(Q\left(G_{1}\right)-V\left(G_{1}\right)\right)}} \sum_{x_{1} x_{2} \in E_{G_{2}}}\left[d\left(t_{1}, x_{1}\right)+d\left(t_{2}, x_{2}\right)\right]
\end{aligned}
$$

$$
\begin{gathered}
=\sum_{\substack{t_{1} t_{2} \in E\left(Q\left(G_{1}\right)\right) \\
t_{1}, t_{2} \in V\left(Q\left(G_{1}\right)-V\left(G_{1}\right)\right)}} \sum_{x_{1} x_{2} \in E_{G_{2}}}\left[d\left(t_{1}, x_{1}\right)+d\left(t_{2}, x_{2}\right)\right]=2\left(n_{2}-1\right) \sum_{\substack{t_{1} t_{2} \in E\left(Q\left(G_{1}\right)\right) \\
t_{1}, t_{2} \in V\left(Q\left(G_{1}\right)-V\left(G_{1}\right)\right)}}\left[d_{\mathrm{QG}_{1}}\left(t_{1}\right)+d_{\mathrm{QG}_{1}}\left(t_{2}\right)\right] . \\
\end{gathered}
$$




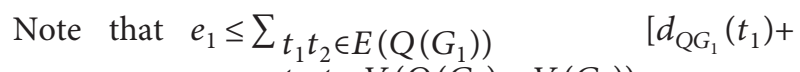
$\left.d_{\mathrm{QG}_{1}}\left(t_{2}\right)\right] \leq M_{1}\left(\mathrm{Q}\left(G_{1}\right)\right)$, so

$$
\begin{aligned}
2\left(n_{2}-1\right) e_{1} & \leq \sum_{4} \leq 2\left(n_{2}-1\right) M_{1}\left(Q\left(G_{1}\right)\right), \\
\sum A_{5} & =\sum_{\substack{t_{1} t_{2} \in E\left(Q\left(G_{1}\right)\right) \\
t_{1}, t_{2} \in V\left(Q\left(G_{1}\right)-V\left(G_{1}\right)\right)}} \sum_{x_{1} x_{2} \notin E_{G_{2}}}\left[d\left(t_{1}, x_{1}\right)+d\left(t_{2}, x_{2}\right)\right] \\
& =\sum_{\substack{t_{1} t_{2} \in E\left(Q\left(G_{1}\right)\right) \\
t_{1}, t_{2} \in V\left(Q\left(G_{1}\right)-V\left(G_{1}\right)\right)}} \sum_{\substack{x_{1} x_{2} \notin E_{G_{2}}\\
}}\left[d\left(t_{1}, x_{1}\right)+d\left(t_{2}, x_{2}\right)\right]=2 \overline{e_{2}} \sum_{\substack{t_{1} t_{2} \in E\left(Q\left(G_{1}\right)\right) \\
t_{1}, t_{2} \in V\left(Q\left(G_{1}\right)-V\left(G_{1}\right)\right)}}\left[d_{Q_{G_{1}}}\left(t_{1}\right)+d_{\mathrm{QG}_{1}}\left(t_{2}\right)\right] .
\end{aligned}
$$

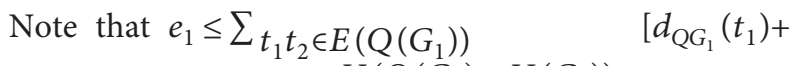
$\left.d_{\mathrm{QG}_{1}}\left(t_{2}\right)\right] \leq M_{1}\left(\mathrm{Q}\left(G_{1}\right)\right)$, so

$$
\begin{aligned}
& 2 \overline{e_{2}} e_{1} \leq \sum A_{5} \leq 2 \overline{e_{2}} M_{1}\left(Q\left(G_{1}\right)\right) \\
& \sum A_{6}=\sum_{\substack{t_{1} t_{2} \notin E\left(Q\left(G_{1}\right)\right) \\
t_{1}, t_{2} \in V\left(Q\left(G_{1}\right)-V\left(G_{1}\right)\right)}} \sum_{x_{1} x_{2} \in E_{G_{2}}}\left[d\left(t_{1}, x_{1}\right)+d\left(t_{2}, x_{2}\right)\right] \\
& =\sum_{\substack{t_{1} t_{2} \notin E\left(Q\left(G_{1}\right)\right) \\
t_{1}, t_{2} \in V\left(Q\left(G_{1}\right)-V\left(G_{1}\right)\right)}} \sum_{x_{1} x_{2} \in E_{G_{2}}}\left[d\left(t_{1}, x_{1}\right)+d\left(t_{2}, x_{2}\right)\right]=2\left(n_{2}-1\right) \sum_{\substack{t_{1} t_{2} \notin E\left(Q\left(G_{1}\right)\right) \\
t_{1}, t_{2} \in V\left(Q\left(G_{1}\right)-V\left(G_{1}\right)\right)}}\left[d_{Q_{G_{1}}}\left(t_{1}\right)+d_{Q_{G_{1}}}\left(t_{2}\right)\right] .
\end{aligned}
$$

Note that $\quad \bar{e}_{1} \leq \sum_{t_{1} t_{2} \notin E\left(Q\left(G_{1}\right)\right)}$

$\left[d_{\mathrm{QG}_{1}}\left(t_{1}\right)+d_{\mathrm{QG}_{1}}\left(t_{2}\right)\right] \leq \bar{M}_{1}\left(\mathrm{Q}\left(G_{1}\right)\right)$, so

$$
t_{1}, t_{2} \in V\left(Q\left(G_{1}\right)-V\left(G_{1}\right)\right)
$$

$$
\begin{aligned}
2\left(n_{2}-1\right) \bar{e}_{1} \leq & \sum A_{6} \leq 2\left(n_{2}-1\right) \bar{M}_{1}\left(Q\left(G_{1}\right)\right), \\
\sum A_{7} & =\sum_{\substack{t_{1} t_{2} \notin E\left(Q\left(G_{1}\right)\right) \\
t_{1}, t_{2} \in V\left(Q\left(G_{1}\right)-V\left(G_{1}\right)\right)}} \sum_{\substack{x_{1} x_{2} \notin E_{G_{2}}\\
}}\left[d\left(t_{1}, x_{1}\right)+d\left(t_{2}, x_{2}\right)\right] \\
& =\sum_{\substack{t_{1} t_{2} \notin E\left(Q\left(G_{1}\right)\right) \\
t_{1}, t_{2} \in V\left(Q\left(G_{1}\right)-V\left(G_{1}\right)\right)}}\left[d\left(t_{1}, x_{1}\right)+d\left(t_{2}, x_{2}\right)\right]=2 \overline{e_{2}} \sum_{\substack{t_{1} t_{2} \notin E\left(Q\left(G_{1}\right)\right) \\
t_{1}, t_{2} \in V\left(Q\left(G_{1}\right)-V\left(G_{1}\right)\right)}}\left[d_{\mathrm{QG}_{1}}\left(t_{1}\right)+d_{\mathrm{QG}_{1}}\left(t_{2}\right)\right] .
\end{aligned}
$$

Note that $\quad \bar{e}_{1} \leq \sum_{t_{1} t_{2} \notin E\left(Q\left(G_{1}\right)\right)}$ $t_{1}, t_{2} \in V\left(Q\left(G_{1}\right)-V\left(G_{1}\right)\right)$ $\left[d_{\mathrm{QG}_{1}}\left(t_{1}\right)+d_{\mathrm{QG}_{1}}\left(t_{2}\right)\right] \leq \bar{M}_{1}\left(\mathrm{Q}\left(G_{1}\right)\right)$, so

$$
2 \overline{e_{2}} \bar{e}_{1} \leq \sum A_{7} \leq 2 \overline{e_{2}} \bar{M}_{1}\left(Q\left(G_{1}\right)\right) .
$$

Consequently, 


$$
\begin{aligned}
& 2\left(n_{2}-1\right) e_{1}+2 \overline{e_{2}} e_{1}+2\left(n_{2}-1\right) e_{1}+2 \overline{e_{2}} e_{1}+2\left(n_{2}-1\right) \bar{e}_{1}+2 \overline{e_{2}} \bar{e}_{1} \\
& \leq \sum A \leq \\
&+n_{2} \bar{M}_{1}\left(Q\left(G_{1}\right)\right)+4\left(n_{2}-1\right) E\left(Q\left(G_{1}\right)\right)+4 \overline{e_{2}} E\left(Q\left(G_{1}\right)\right)+2\left(n_{2}-1\right) M_{1}\left(Q\left(G_{1}\right)\right) \\
&+2 \bar{e}_{2} M_{1}\left(Q\left(G_{1}\right)\right)+2\left(n_{2}-1\right) \bar{M}_{1}\left(Q\left(G_{1}\right)\right)+2{\overline{e_{2}}}_{M_{1}}\left(Q\left(G_{1}\right)\right) .
\end{aligned}
$$

Using equation (8), we directly have

$$
\begin{aligned}
& \sum B=4 e_{1} \bar{e}_{2}+e_{2}\left(e_{1}+\bar{e}_{1}\right)+\left(2\left(e_{2}+\bar{e}_{2}\right)+n_{2}\right) M_{1}\left(G_{1}\right)+\left(n_{2}\left(2 e_{2}+\bar{e}_{2}\right)\right) \bar{M}_{1}\left(G_{1}\right) \\
& +2\left(e_{2}+\bar{e}_{1}\right) M_{1}\left(G_{2}\right)+n_{1}+2\left(\bar{e}_{1}+e_{1}\right) \bar{M}_{1}\left(G_{2}\right), \\
& \sum C=\sum C_{1}+\sum C_{2}+\sum C_{3} \text {, } \\
& \sum C_{1}=\sum_{\substack{t_{1} t_{2} \notin E\left(Q\left(G_{1}\right)\right) \\
t_{1} \in V\left(G_{1}\right) \\
t_{2} \in V\left(Q\left(G_{1}\right)-V\left(G_{1}\right)\right)}} \sum_{x \in V_{G_{2}}}\left[d\left(t_{1}, x\right)+d\left(t_{2}, x\right)\right]=\sum_{\substack{t_{1} t_{2} \notin E\left(Q\left(G_{1}\right)\right) \\
t_{1} \in V\left(G_{1}\right) \\
t_{2} \in V\left(Q\left(G_{1}\right)-V\left(G_{1}\right)\right)}} \sum_{x \in V_{G_{2}}}\left[d_{G_{1}}\left(t_{1}\right)+d(x)+d_{Q\left(G_{1}\right)}\left(t_{2}\right)\right] \\
& \begin{aligned}
=n_{2} \sum_{\substack{t_{1} t_{2} \notin E\left(Q\left(G_{1}\right)\right) \\
t_{1} \in V\left(G_{1}\right) \\
t_{2} \in V\left(Q\left(G_{1}\right)-V\left(G_{1}\right)\right)}} d_{Q\left(G_{1}\right)}\left(t_{1}\right)+2 e_{2} e_{1}\left(n_{1}-2\right)+3 n_{2} e_{1}\left(n_{1}-2\right) . \\
\end{aligned}
\end{aligned}
$$

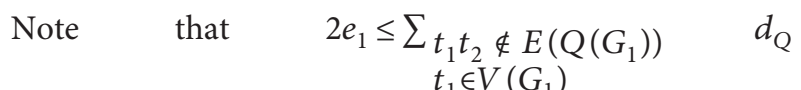

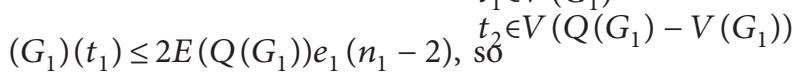

$$
\begin{aligned}
& 2 n_{2} e_{1}+2 e_{2} e_{1}\left(n_{1}-2\right)+3 n_{2} e_{1}\left(n_{1}-2\right) \leq \sum C_{1} \leq 2 n_{2} E\left(Q\left(G_{1}\right)\right) e_{1}\left(n_{1}-2\right)+2 e_{2} e_{1}\left(n_{1}-2\right)+3 n_{2} e_{1}\left(n_{1}-2\right), \\
& \sum C_{2}=\sum_{t_{1} t_{2} \notin E\left(Q\left(G_{1}\right)\right)} \sum_{\left(x_{1}, x_{2}\right) \in V_{G_{2}}}\left[d\left(t_{1}, x_{1}\right)+d\left(t_{2}, x_{2}\right)\right] \\
& t_{1} \in V\left(G_{1}\right) \\
& t_{2} \in V\left(Q\left(G_{1}\right)-V\left(G_{1}\right)\right) \\
& \sum C_{2}=\sum_{\substack{t_{1} t_{2} \notin E\left(Q\left(G_{1}\right)\right) \\
t_{1} \in V\left(G_{1}\right) \\
t_{2} \in V\left(Q\left(G_{1}\right)-V\left(G_{1}\right)\right)}} \sum_{\substack{x_{1}, x_{2} \in V_{G_{2}}\\
}}\left[d_{Q\left(G_{1}\right)}\left(t_{1}\right)+d\left(x_{1}\right)+d_{Q\left(G_{1}\right)}\left(t_{2}\right)\right] \\
& =n_{2}\left(n_{2}-1\right) \sum_{\substack{t_{1} t_{2} \notin E\left(Q\left(G_{1}\right)\right) \\
t_{1} \in V\left(G_{1}\right) \\
\\
t_{2} \in V\left(Q\left(G_{1}\right)-V\left(G_{1}\right)\right)}} d_{Q\left(G_{1}\right)}\left(t_{1}\right)+\left(2 e_{2}+3 n_{2}\right)\left(n_{2}-1\right) \mid \\
& \sum_{t_{1} t_{2} \notin E\left(Q\left(G_{1}\right)\right)} d_{Q\left(G_{1}\right)}\left(t_{1}\right) \mid \text {. }
\end{aligned}
$$




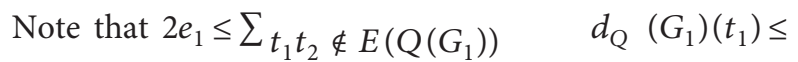

$$
t_{1} \in V\left(G_{1}\right)
$$

$2 E\left(Q\left(G_{1}\right)\right) e_{1}\left(n_{1}-2\right)$, so

$$
t_{2} \in V\left(Q\left(G_{1}\right)-V\left(G_{1}\right)\right)
$$

$$
\begin{aligned}
& 2 e_{1} n_{2}\left(n_{2}-1\right)+e_{1}\left(n_{1}-2\right)\left[\left(2 e_{2}+3 n_{2}\right)\left(n_{2}-1\right)\right] \leq \sum C_{2} \leq 2 E\left(Q\left(G_{1}\right)\right) e_{1}\left(n_{1}-2\right)\left[n_{2}\left(n_{2}-1\right)+\left(2 e_{2}+3 n_{2}\right)\left(n_{2}-1\right)\right] \\
& \sum C_{3}=\sum_{\substack{t_{1} t_{2} \in E\left(Q\left(G_{1}\right)\right) \\
t_{1} \in\left(G_{1}\right) \\
t_{2} \in V\left(Q\left(G_{1}\right)-V\left(G_{1}\right)\right)}} \sum_{x_{1}, x_{2} \in V_{G_{2}}}\left[d\left(t_{1}, x_{1}\right)+d\left(t_{2}, x_{2}\right)\right] \\
& =\sum_{\substack{t_{1} t_{2} \in E\left(Q\left(G_{1}\right)\right) \\
t_{1} \in V\left(G_{1}\right) \\
t_{2} \in V\left(Q\left(G_{1}\right)-V\left(G_{1}\right)\right)}} \sum_{\substack{x_{1}, x_{2} \in V_{G_{2}} \\
t_{2}}}\left[d_{Q\left(G_{1}\right)}\left(t_{1}\right)+d\left(x_{1}\right)+d_{Q\left(G_{1}\right)}\left(t_{2}\right)\right] \\
& =n_{2}\left(n_{2}-1\right) \sum_{\substack{t_{1} t_{2} \in E\left(Q\left(G_{1}\right)\right) \\
t_{1} \in V\left(G_{1}\right) \\
t_{2} \in V\left(Q\left(G_{1}\right)-V\left(G_{1}\right)\right)}} d_{Q\left(G_{1}\right)}\left[\left(t_{1}\right)\right]+2 e_{2}\left(n_{2}-1\right) \mid
\end{aligned}
$$

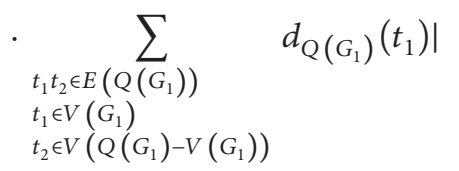

$$
\begin{aligned}
& +3 n_{2}\left(n_{2}-1\right)\left|\sum_{\substack{t_{1} t_{2} \in E\left(Q\left(G_{1}\right)\right) \\
t_{1} \in V\left(G_{1}\right) \\
t_{2} \in V\left(Q\left(G_{1}\right)-V\left(G_{1}\right)\right)}} d_{Q\left(G_{1}\right)}\left[\left(t_{1}\right)\right]\right|
\end{aligned}
$$

Note that $2 E\left(G_{1}\right) \leq \sum_{t_{1} t_{2} \in E\left(Q\left(G_{1}\right)\right) \quad d_{Q}}$

$\left(G_{1}\right)\left[\left(t_{1}\right)\right] \leq 4 E\left(Q\left(G_{1}\right)\right) e_{1}$, so $\quad t_{2} \in V\left(Q\left(G_{1}\right)-V\left(G_{1}\right)\right)$

$$
4 n_{2}\left(n_{2}-1\right) e_{1}+\left(n_{2}-1\right)\left(2 e_{1}\right)\left(2 e_{2}\left(2 e_{1}\right)+3 n_{2}\right) \leq \sum C_{3} \leq 4 n_{2}\left(n_{2}-1\right) Q\left(E\left(G_{1}\right)\right) e_{1}+\left(n_{2}-1\right)\left(2 e_{1}\right)\left(2 e_{2}+3 n_{2}\right) .
$$

Consequently,

$$
\begin{aligned}
2 n_{2} e_{1}+2 e_{2} e_{1}\left(n_{1}-2\right)+3 n_{2} e_{1}\left(n_{1}-2\right)+2 e_{1} n_{2}\left(n_{2}-1\right)+e_{1}\left(n_{1}-2\right)\left[\left(2 e_{2}+3 n_{2}\right)\left(n_{2}-1\right)\right] \\
\quad+4 n_{2}\left(n_{2}-1\right) e_{1}+\left(n_{2}-1\right)\left(2 e_{1}\right)\left(2 e_{2}\left(2 e_{1}\right)+3 n_{2}\right) \\
\leq \sum C \leq \\
\quad 2 n_{2} E\left(Q\left(G_{1}\right)\right) e_{1}\left(n_{1}-2\right)+2 e_{2} e_{1}\left(n_{1}-2\right)+3 n_{2} e_{1}\left(n_{1}-2\right)+2 E\left(Q\left(G_{1}\right)\right) e_{1}\left(n_{1}-2\right)\left[n_{2}\left(n_{2}-1\right)+\left(2 e_{2}+3 n_{2}\right)\left(n_{2}-1\right)\right] \\
\quad+4 n_{2}\left(n_{2}-1\right) Q\left(E\left(G_{1}\right)\right) e_{1}+\left(n_{2}-1\right)\left(2 e_{1}\right)\left(2 e_{2}+3 n_{2}\right) .
\end{aligned}
$$

By putting the values of $\sum A+\sum B+\sum C$ in equation (25), we get the required proof.
Theorem 4. Let $G_{1}$ and $G_{2}$ be two connected graphs; then, first Zagreb coindex of $G_{1+T} G_{2}$ is given as 


$$
\alpha_{1} \leq \bar{M}\left(G_{1+T} G_{2}\right) \leq \alpha_{2},
$$

where

$$
\begin{aligned}
\alpha_{1}= & 4\left(2 e_{1} \bar{e}_{2}+e_{2} \bar{e}_{1}\right)+e_{1}\left(n_{1}-2\right)\left(2 e_{2}+3 n_{2}\right)+2\left(n_{2}+2\left(e_{2}+\bar{e}_{2}\right)\right) M_{1}\left(G_{1}\right)+4\left(e_{2}+\bar{e}_{2}\right) \bar{M}_{1}\left(G_{1}\right)+2\left(e_{1}+\bar{e}_{1}\right) M_{1}\left(G_{2}\right) \\
& +\left(n_{1}+2\left(\bar{e}_{1}+e_{1}\right)\right) \bar{M}_{1}\left(G_{2}\right)+2\left(\left(n_{2}-1\right)+\overline{e_{2}}\right) e_{1}+\left(n_{2}-1+\overline{e_{2}}\right) e_{1} \bar{e}_{1}+2 n_{2}^{2} e_{1}+2 e_{1}\left(n_{2}-1\right)\left[n_{2}+2 e_{2}+3 n_{2}\right], \\
\alpha_{2}= & 4\left(2 e_{1} \bar{e}_{2}+e_{2} \bar{e}_{1}\right)+e_{1}\left(n_{1}-2\right)\left(2 e_{2}+3 n_{2}\right)+4\left(e_{2}+\bar{e}_{2}\right) M_{1}\left(G_{1}\right)+\left(2 n_{2}+4\left(e_{2}+\bar{e}_{2}\right)\right) \bar{M}_{1}\left(G_{1}\right) \\
& +2\left(e_{1}+\bar{e}_{1}\right) M_{1}\left(G_{2}\right)+\left(n_{1}+2\left(\bar{e}_{1}+e_{1}\right)\right) \bar{M}_{1}\left(G_{2}\right)+n_{2} \bar{M}_{1} T\left(G_{1}\right)+4\left(\left(n_{2}-1\right)+\overline{m_{2}}\right) E T\left(G_{1}\right) \\
& +\left(n_{2}-1+\overline{m_{2}}\right) M_{1}\left(T\left(G_{1}\right)\right) \bar{M}_{1}\left(T\left(G_{1}\right)\right)+n_{2}^{2} E\left(T\left(G_{1}\right)\right) e_{1}\left(n_{2}-1\right)+2 e_{1}\left(2 e_{2}+3 n_{2}\right)\left(n_{2}-1\right) \\
& +4 n_{2} e_{1} E T\left(G_{1}\right)\left(n_{2}-1\right)+2 e_{1}\left(n_{2}-1\right)\left(2 e_{2}+3 n_{2}\right) .
\end{aligned}
$$

Proof. Using equation (2), we have

$$
\begin{aligned}
\bar{M}\left(G_{1+T} G_{2}\right)= & \sum_{\left(u_{1}, u_{2}\right)\left(v_{1}, v_{2}\right) \notin E\left(G_{1+T} G_{2}\right)}\left[d\left(u_{1}, v_{1}\right)+d\left(u_{2}, v_{2}\right)\right], \\
\bar{M}\left(G_{1+T} G_{2}\right)= & \sum_{t_{1}, t_{2} \in V} \sum_{\left(T\left(G_{1}\right)-V\left(G_{1}\right)\right)}\left[d\left(t_{1}, x_{1}\right)+d\left(t_{2}, x_{2}\right)\right]+\sum_{t_{1}, x_{2} \in V_{G_{1}} \in V_{G_{2}}} \sum_{x_{1}, x_{2} \in V_{G_{2}}}\left[d\left(t_{1}, x_{1}\right)+d\left(t_{2}, x_{2}\right)\right] \\
& +\sum_{t_{1}, t_{2} \in V} \sum_{\left(T\left(G_{1}\right)-V\left(G_{1}\right)\right)} \sum_{x_{1}, x_{2} \in V_{G_{2}}}\left[d\left(t_{1}, x_{1}\right)+d\left(t_{2}, x_{2}\right)\right] \\
= & \sum A+\sum B+\sum C .
\end{aligned}
$$

Using equations (30) and (17), we directly have

$$
\begin{aligned}
2\left(n_{2}-1\right) e_{1}+2 \overline{e_{2}} e_{1}+2\left(n_{2}-1\right) e_{1}+2 \overline{e_{2}} e_{1}+2\left(n_{2}-1\right) \bar{e}_{1}+2 \overline{e_{2}} \bar{e}_{1} \leq & \sum A \leq+n_{2} \bar{M}_{1}\left(Q\left(G_{1}\right)\right)+4\left(n_{2}-1\right) E\left(Q\left(G_{1}\right)\right) \\
& +4 \overline{e_{2}} E\left(Q\left(G_{1}\right)\right)+2\left(n_{2}-1\right) M_{1}\left(Q\left(G_{1}\right)\right)+2 \overline{e_{2}} M_{1}\left(Q\left(G_{1}\right)\right) \\
& +2\left(n_{2}-1\right) \bar{M}_{1}\left(Q\left(G_{1}\right)\right)+2{\overline{e_{2}}}_{1} \bar{M}_{1}\left(Q\left(G_{1}\right)\right) \\
\sum B= & 4\left(2 e_{1} \bar{e}_{2}+4 \bar{e}+e_{2} \bar{e}_{1}\right)+\left(4 e_{2}+4 \bar{e}_{2}\right) M_{1}\left(G_{1}\right) \\
& +2\left(e_{1}+\bar{e}_{1}\right) M_{1}\left(G_{2}\right)+2 n_{2}+2\left(e_{2}+\bar{e}_{2}\right) \bar{M}_{1}\left(G_{1}\right) \\
& +\left(n_{1}+2 \bar{e}_{1}+2 e_{1}\right) \bar{M}_{1}\left(G_{2}\right) .
\end{aligned}
$$


Now, we find

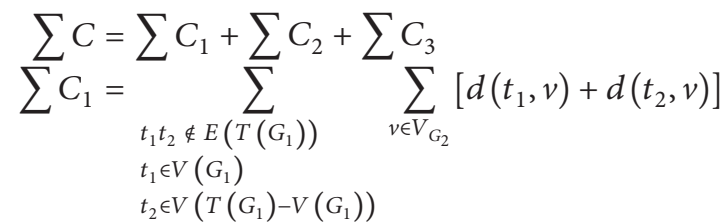

$$
\begin{aligned}
& 2 n_{2} \bar{e}_{1}+2 e_{2} e_{1}\left(n_{1}-2\right) 3 n_{2} e_{1}\left(n_{1}-2\right) \leq \sum C_{1} \leq 2 n_{2} T\left(E\left(G_{1}\right)\right) e_{1}\left(n_{1}-2\right)+2 e_{2} e_{1}\left(n_{1}-2\right)+3 n_{2} e_{1}\left(n_{1}-2\right) \\
& \sum C_{2}=\sum_{t_{1} t_{2} \notin E\left(T\left(G_{1}\right)\right)} \sum_{\left(x_{1}, x_{2}\right) \in V_{G_{2}}}\left[d\left(t_{1}, x_{1}\right)+d\left(t_{2}, x_{2}\right)\right] \\
& t_{1} \in V\left(G_{1}\right) \\
& t_{2} \in V\left(T\left(G_{1}\right)-V\left(G_{1}\right)\right) \\
& 2 n_{2}\left(n_{2}-1\right) e_{1}+2\left(2 e_{2}+3 n_{2}\right)\left(n_{2}-1\right) e_{1}\left(n_{1}-2\right) \leq \sum C_{2} \leq 2 n_{2}\left(n_{2}-1\right) E\left(T\left(G_{1}\right)\right) e_{1}\left(n_{1}-2\right) a \\
& +2\left(2 e_{2}+3 n_{2}\right)\left(n_{2}-1\right) T\left(E\left(G_{1}\right)\right) e_{1}\left(n_{1}-2\right) \\
& \sum C_{3}=\sum_{t_{1} t_{2} \in E\left(T\left(G_{1}\right)\right)} \sum_{x_{1}, x_{2} \in V_{G_{2}}}\left[d\left(t_{1}, x_{1}\right)+d\left(t_{2}, x_{2}\right)\right] \\
& t_{1} \in V\left(G_{1}\right) \\
& t_{2} \in V\left(T\left(G_{1}\right)-V\left(G_{1}\right)\right) \\
& 4 n_{2} e_{1}\left(n_{2}-1\right)+4 e_{1} e_{2}\left(n_{2}-1\right)+4 e_{1}\left(3 n_{2}\left(n_{2}-1\right)\right) T\left(E\left(G_{1}\right)\right) \\
& \leq \sum C_{3} \leq 8 n_{2} e_{1}\left(n_{2}-1\right) T\left(E\left(G_{1}\right)\right) \\
& +8 e_{1} e_{2}\left(n_{2}-1\right) T\left(E\left(G_{1}\right)\right)+4 e_{1}\left(3 n_{2}\left(n_{2}-1\right)\right) T\left(E\left(G_{1}\right)\right) \text {. }
\end{aligned}
$$

Consequently,

$$
\begin{aligned}
& 2 n_{2} \bar{e}_{1}+2 e_{2} e_{1}\left(n_{1}-2\right)+3 n_{2} e_{1}\left(n_{1}-2\right)+2 n_{2}\left(n_{2}-1\right) e_{1}+2\left(2 e_{2}+3 n_{2}\right)\left(n_{2}-1\right) e_{1}\left(n_{1}-2\right)+4 n_{2} e_{1}\left(n_{2}-1\right)+4 e_{1} e_{2}\left(n_{2}-1\right) \\
& \quad+4 e_{1}\left(3 n_{2}\left(n_{2}-1\right)\right) T\left(E\left(G_{1}\right)\right) \\
& \leq \\
& \quad \sum C \leq \\
& \quad 2 n_{2} T\left(E\left(G_{1}\right)\right) e_{1}\left(n_{1}-2\right)+2 e_{2} e_{1}\left(n_{1}-2\right)+3 n_{2} e_{1}\left(n_{1}-2\right)+2 n_{2}\left(n_{2}-1\right) E\left(T\left(G_{1}\right)\right) e_{1}\left(n_{1}-2\right) \\
& \quad+2\left(2 e_{2}+3 n_{2}\right)\left(n_{2}-1\right) T\left(E\left(G_{1}\right)\right) e_{1}\left(n_{1}-2\right)+8 n_{2} e_{1}\left(n_{2}-1\right) T\left(E\left(G_{1}\right)\right) \\
& \quad+8 e_{1} e_{2}\left(n_{2}-1\right) T\left(E\left(G_{1}\right)\right)+4 e_{1}\left(3 n_{2}\left(n_{2}-1\right)\right) T\left(E\left(G_{1}\right)\right) .
\end{aligned}
$$

By putting the values of $\sum A+\sum B+\sum C$ in equation (39), we get the required proof.

\section{Conclusion}

Several topological indices (TIs) have been defined with the help of the graph-theoretic parameters of degree, distance, eccentricity, and eigenvalues, and various results are obtained by different researchers for a large number of molecular structures, graphs, or networks. This popularity is not only due to being the rich mathematical problems of the theory of graphs and networking but also due to the wide range of its applications in different disciplines of sciences, especially in chemistry and computer science such as navigation, combinatorial optimization, pattern recognition, image processing, integer programming, formation of chemical compounds, and discovery of drugs.

In this paper, we have computed bounds for first Zagreb coindex of $F$ - sum graphs such as $\bar{M}\left(G_{1+S} G_{2}\right), \bar{M}\left(G_{1+R} G_{2}\right)$, $\bar{M}\left(G_{1+Q} G_{2}\right)$, and $\bar{M}\left(G_{1+T} G_{2}\right)$ in their general forms. The $F$-sum graphs obtained by different subdivision operations and Cartesian product of graphs present several families of molecular structures, especially graphs consisting of hexagonal chains.

Table 1 presents the first Zagreb coindex of the Cartesian product of certain path graphs, and Tables 2-5 show the lower and upper bounds of the first Zagreb coindex for the $S$-sum, $R$-sum, Q-sum, and T-sum graphs. Now, we compute the linear regression formula to compute correlation between exact values of $\left(\bar{M}_{1}\left(P_{n}+P_{m}\right)\right)$ and bounded values 
TABLE 1: Exact value of first Zagreb coindex for Cartesian product of path graphs.

\begin{tabular}{lc}
\hline Cartesian product of paths & Exact value of $\overline{M_{1}}$ \\
\hline$P_{2} \otimes P_{2}$ & 36 \\
$P_{3} \otimes P_{3}$ & 374 \\
$P_{4} \otimes P_{4}$ & 1748 \\
$P_{5} \otimes P_{5}$ & 4853 \\
\hline
\end{tabular}

TABLe 2: Bounds of first Zagreb coindex for S-sum graph.

\begin{tabular}{lcc}
\hline$S$-sum graphs & Lower bound of $\overline{M_{1}}$ & Upper bound of $\overline{M_{1}}$ \\
\hline$P_{2+S} P_{2}$ & 36 & 40 \\
$P_{3+S} P_{3}$ & 374 & 534 \\
$P_{4+S} P_{4}$ & 1748 & 3284 \\
$P_{5+S} P_{5}$ & 4853 & 10176 \\
\hline
\end{tabular}

TABle 3: Bounds of first Zagreb coindex for $R$-sum graph.

\begin{tabular}{lcc}
\hline$R$-sum graphs & Lower bound of $\overline{M_{1}}$ & Upper bound of $\overline{M_{1}}$ \\
\hline$P_{2+R} P_{2}$ & 36 & 44 \\
$P_{3+R} P_{3}$ & 510 & 1430 \\
$P_{4+R} P_{4}$ & 2208 & 7584 \\
$P_{5+R} P_{5}$ & 7314 & 27442 \\
\hline
\end{tabular}

TABle 4: Bounds of first Zagreb coindex for Q-sum graph.

\begin{tabular}{lcc}
\hline$Q$-sum operation & Lower bound of $\overline{M_{1}}$ & Upper bound of $\overline{M_{1}}$ \\
\hline$P_{2+Q} P_{2}$ & 36 & 92 \\
$P_{3+Q} P_{3}$ & 470 & 2500 \\
$P_{4+Q} P_{4}$ & 1756 & 6708 \\
$P_{5+Q} P_{5}$ & 9166 & 22409 \\
\hline
\end{tabular}

TABLE 5: Bounds of first Zagreb coindex for T-sum graph.

\begin{tabular}{lcc}
\hline$T$-sum operation & Lower bound of $\overline{M_{1}}$ & Upper bound of $\overline{M_{1}}$ \\
\hline$P_{2+T} P_{2}$ & 38 & 118 \\
$P_{3+T} P_{3}$ & 556 & 4370 \\
$P_{4+T} P_{4}$ & 2004 & 10488 \\
$P_{5+T} P_{5}$ & 9842 & 12807 \\
\hline
\end{tabular}

of F-sum graphs $\left(\bar{M}_{1}\left(P_{n_{F}}+P_{m}\right)\right)$. In general, the linear regression formula is expressed as $Y=b_{0}+b_{1} X$, where $b_{0}$ is the $y$-intercept (point of intersection of line and $Y$-axis) and $b_{1}$ is slope that describes the line's direction and inclination.

The linear regression formula of the Cartesian product of paths $Y$ in terms of lower and upper bounds of the $S$-sum graph $(X)$ is $Y=0+1 X$ (100 percent correlation between $x$ and $y$ exists with 1 being the value of correlation coefficient) and $Y=103.9108+0.47 X$ (a very strong direct relationship between $X$ and $Y$ exists with 0.9993 being the value of correlation coefficient).

The linear regression formula of the Cartesian product of paths $Y$ in terms of lower and upper bounds of the $R$-sum graph $(X)$ is $Y=96.9504+0.6578 X$ (a very strong direct relationship between $X$ and $Y$ exists with 0.9982 being the value of correlation coefficient) and $Y=173.5697+0.1731 X$ (a very strong direct relationship between $X$ and $Y$ exists with 0.9966 being the value of correlation coefficient).

The linear regression formula of the Cartesian product of paths $Y$ in terms of lower and upper bounds of the $Q$-sum graph $(X)$ is $Y=305.2811+0.5066 X$ (a very strong direct relationship between $X$ and $Y$ exists with 0.9851 being the value of correlation coefficient) and $Y=24.8959+0.218 X$ (a very strong direct relationship between $X$ and $Y$ exists with 0.9962 being the value of correlation coefficient).

The linear regression formula of the Cartesian product of paths $Y$ in terms of lower and upper bounds of the $T$-sum graph $(X)$ is $Y=276.3798+0.4747 X$ (a very strong direct relationship between $X$ and $Y$ exists with 0.9870 being the value of correlation coefficient) and $Y=-565.4512+0.3338 X$ (a very strong direct relationship between $X$ and $Y$ exists with 0.8784 being the value of correlation coefficient).

Now, we close our discussion with the comment that the molecular structures which are isomorphic to sum graphs ( $S$-sum, $R$-sum, $Q$-sum, and $T$-sum) have strong correlation for the thirteen physicochemical properties such as boiling point, density, heat capacity at constant pressure, entropy, heat capacity at constant time, enthalpy of vaporization, acentric factor, standard enthalpy of vaporization, enthalpy of formation, octanol-water partition coefficient, standard enthalpy of formation, total surface area, and molar volume.

\section{Data Availability}

All data are included within this article. However, the reader may contact the corresponding author for more details of the data.

\section{Conflicts of Interest}

The authors declare that they have no conflicts of interest.

\section{Acknowledgments}

This study was supported by Natural Science Fund of Education Department of Anhui Province under grant no. KJ2020A0478.

\section{References}

[1] G. Rcker and C. Rcker, "On topological indices, boiling points, and cycloalkanes," Journal of Chemical Information and Computer Sciences, vol. 39, pp. 788-802, 1999.

[2] A. R. Matamala and E. Estrada, "Generalised topological indices: optimisation methodology and physico-chemical interpretation," Chemical Physics Letters, vol. 410, no. 4-6, pp. 343-347, 2005.

[3] H. Gonzalez-Diaz, S. Vilar, L. Santana, and E. Uriarte, "Medicinal chemistry and bioinformatics - current trends in drugs discovery with networks topological indices," Current Topics in Medicinal Chemistry, vol. 7, no. 10, pp. 1015-1029, 2007. 
[4] W. Yan, B.-Y. Yang, and Y.-N. Yeh, "The behavior of Wiener indices and polynomials of graphs under five graph decorations," Applied Mathematics Letters, vol. 20, no. 3, pp. 290-295, 2007.

[5] M. V. Diudea, QSPR/QSAR Studies by Molecular Descriptors, Nova Science Publishers, Hauppaauge, NY, USA, 2001.

[6] I. Gutman, "Degree-based topological indices," Croatica Chemica Acta, vol. 86, no. 4, pp. 351-361, 2013.

[7] H. Wiener, "Structural determination of paraffin boiling points," Journal of the American Chemical Society, vol. 69, no. 1, pp. 17-20, 1947.

[8] I. Gutman and N. Trinajstić, "Graph theory and molecular orbitals. Total $\varphi$-electron energy of alternant hydrocarbons," Chemical Physics Letters, vol. 17, no. 4, pp. 535-538, 1972.

[9] M. Eliasi and B. Taeri, "Four new sums of graphs and their Wiener indices," Discrete Applied Mathematics, vol. 157, no. 4, pp. 794-803, 2009.

[10] S. Akhter and M. Imran, "Computing the forgotten topological index of four operations on graphs," $A K C E$ International Journal of Graphs and Combinatorics, vol. 14, no. 1, pp. 70-79, 2017.

[11] H. Deng, D. Sarala, S. K. Ayyaswamy, and S. Balachandran, "The Zagreb indices of four operations on graphs," Applied Mathematics and Computation, vol. 275, pp. 422-431, 2016.

[12] J.-B. Liu, S. Javed, M. Javaid, and K. Shabbir, "Computing first general Zagreb index of operations on graphs," IEEE Access, vol. 7, pp. 47494-47502, 2019.

[13] A. R. Ashrafi, T. Došlić, and A. Hamzeh, "The Zagreb coindices of graph operations," Discrete Applied Mathematics, vol. 158, no. 15, pp. 1571-1578, 2010.

[14] T. Mansour and C. Song, "The a and (a, b) analogs of Zagreb Indices and coindices of graphs," International Journal of Combinatorics, vol. 2012, Article ID 909285, 10 pages, 2012.

[15] A. R. Ashrafi, T. Došlic, and A. Hamzeh, "Extremal graphs with respect to the Zagreb coindices," MATCH Communications in Mathematical and in Computer Chemistry, vol. 5, no. 1, pp. 85-92, 2011.

[16] H. S. Ramane, S. Y. Talwar, and I. N. Cangul, "Transmission and reciprocal transmission based topological Co-indices of graphs," European Journal of Pure and Applied Mathematics, vol. 13, no. 5, pp. 1057-1071, 2020.

[17] M. Javaid, U. Ali, and J. B. Liu, "Computing analysis for first Zagreb connection index and coindex of resultant graphs," Mathematical Problems in Engineering, vol. 2021, Article ID 6019517, 19 pages, 2021. 